\title{
EXACT CATEGORIES AND VECTOR SPACE CATEGORIES
}

\author{
PETER DRÄXLER, IDUN REITEN, SVERRE O. SMALØ, ØYVIND SOLBERG, \\ AND WITH AN APPENDIX BY B. KELLER
}

\begin{abstract}
In a series of papers additive subbifunctors $F$ of the bifunctor $\operatorname{Ext}_{\Lambda}($,$) are studied in order to establish a relative homology theory for an$ artin algebra $\Lambda$. On the other hand, one may consider the elements of $F(X, Y)$ as short exact sequences. We observe that these exact sequences make $\bmod \Lambda$ into an exact category if and only if $F$ is closed in the sense of Butler and Horrocks.

Concerning the axioms for an exact category we refer to Gabriel and Roiter's book. In fact, for our general results we work with subbifunctors of the extension functor for arbitrary exact categories.

In order to study projective and injective objects for exact categories it turns out to be convenient to consider categories with almost split exact pairs, because many earlier results can easily be adapted to this situation.

Exact categories arise in representation theory for example if one studies categories of representations of bimodules. Representations of bimodules gained their importance in studying questions about representation types. They appear as domains of certain reduction functors defined on categories of modules. These reduction functors are often closely related to the functor $\operatorname{Ext}_{\Lambda}($,$) and in general do not preserve at all the usual exact structure of$ $\bmod \Lambda$.

By showing the closedness of suitable subbifunctors of $\operatorname{Ext}_{\Lambda}($,$) we can$ equip $\bmod \Lambda$ with an exact structure such that some reduction functors actually become 'exact'. This allows us to derive information about the projective and injective objects in the respective categories of representations of bimodules appearing as domains, and even show that almost split sequences for them.

Examples of such domains appearing in practice are the subspace categories of a vector space category with bonds. We provide an example showing that existence of almost split sequences for them is not a general fact but may even fail if the vector space category is finite.
\end{abstract}

\section{EXACT CATEGORIES}

This section is devoted to transferring some definitions and basic results on relative theory developed for abelian categories in $[\mathrm{BH}]$ and for finitely generated modules over artin algebras in [ASo] to the context of exact categories. Furthermore, we show how one can construct a new exact structure on a category from closed subbifunctors of the extension bifunctor induced by a given exact structure.

Received by the editors January 27, 1997.

1991 Mathematics Subject Classification. Primary 16B50; Secondary 16G20, 16G70.

Professors Reiten, Smalø and Solberg thank the Norwegian Research Council for partial support during the preparation of this paper.

(C)1999 American Mathematical Society 
Moreover, we study when an exact structure on a category $\mathcal{A}$ induces an exact structure on a factor category of $\mathcal{A}$.

1.1. Axioms for exact categories. Let $\mathcal{A}$ be an additive category with split idempotents. From $[\mathrm{GR}]$ we recall the following notions. A pair $(i, d)$ of composable morphisms $X \stackrel{i}{\rightarrow} Y \stackrel{d}{\rightarrow} Z$ in $\mathcal{A}$ is called exact if $i$ is a kernel of $d$ and $d$ is a cokernel of $i$.

Let $\mathcal{E}$ be a class of exact pairs $X \stackrel{i}{\rightarrow} Y \stackrel{d}{\rightarrow} Z$ which is closed under isomorphisms. The morphisms $i$ and $d$ appearing in a pair $(i, d)$ in $\mathcal{E}$ are called an inflation and a deflation of $\mathcal{E}$, respectively. The class $\mathcal{E}$ is said to be an exact structure on $\mathcal{A}$ and $(\mathcal{A}, \mathcal{E})$ an exact category if the following axioms are satisfied:

E1 The composition of two deflations is a deflation.

E2 For each $f$ in $\mathcal{A}\left(Z^{\prime}, Z\right)$ and each deflation $d$ in $\mathcal{A}(Y, Z)$, there is some $Y^{\prime}$ in $\mathcal{A}$, an $f^{\prime}$ in $\mathcal{A}\left(Y^{\prime}, Y\right)$ and a deflation $d^{\prime}: Y^{\prime} \rightarrow Z^{\prime}$ such that $d f^{\prime}=f d^{\prime}$.

E3 Identities are deflations. If $d e$ is a deflation, then so is $d$.

E3 ${ }^{\text {op }}$ Identities are inflations. If $j i$ is a inflation, then so is $i$.

This set of axioms is proved to be equivalent to the following one (see [Ke2]):

Ex0 The identity morphism of the zero object, $i_{0}$, is a deflation.

Ex1 The composition of two deflations is a deflation.

Ex2 For each $f$ in $\mathcal{A}\left(Z^{\prime}, Z\right)$ and each deflation $d$ in $\mathcal{A}(Y, Z)$, there is a pullback diagram

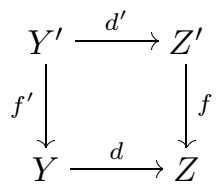

where $d^{\prime}$ is a deflation.

Ex $2^{\text {op }}$ For each $f$ in $\mathcal{A}\left(X, X^{\prime}\right)$ and each inflation $i$ in $\mathcal{A}(X, Y)$, there is a pushout diagram

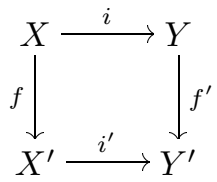

where $i^{\prime}$ is an inflation.

Since the morphisms $d$ and $d^{\prime}$ in Ex2 are deflations, there are corresponding inflations $i: X \rightarrow Y, i^{\prime}: X^{\prime} \rightarrow Y^{\prime}$ such that $(i, d)$ and $\left(i^{\prime}, d^{\prime}\right)$ belong to $\mathcal{E}$. Using the pullback property one sees that $f^{\prime} i^{\prime}$ is a kernel of $d$. Hence by possibly modifying $i^{\prime}$ by a unique isomorphism we may suppose $f^{\prime} i^{\prime}=i$. We call the pair $\left(i^{\prime}, d^{\prime}\right)$ a pullback pair of $(i, d)$ along $f$. Dually, we use the notion of a pushout pair.

It is shown in [Ke1, Appendix $\mathrm{A}]$ that each skeletally small exact category $(\mathcal{A}, \mathcal{E})$ admits an equivalence $F: \mathcal{A} \rightarrow \mathcal{B}$ with a full subcategory $\mathcal{B}$ of an abelian category $\mathcal{C}$, such that $\mathcal{B}$ is extension closed, and an exact pair $(i, d)$ is in $\mathcal{E}$ if and only if

$$
0 \rightarrow F(X) \stackrel{F(i)}{\longrightarrow} F(Y) \stackrel{F(d)}{\longrightarrow} F(Z) \rightarrow 0
$$

is a short exact sequence in $\mathcal{C}$. Moreover, it is established that an exact category also satisfies Quillen's axioms from $[\mathrm{Qu}]$. As a particular consequence, also the dual 
axiom of E1 is satisfied. Thus the duals of all axioms are satisfied. We sum this up in the following proposition, which is used later.

Proposition 1.1. If $(\mathcal{A}, \mathcal{E})$ is an exact category, then also $\left(\mathcal{A}^{\mathrm{op}}, \mathcal{E}^{\mathrm{op}}\right)$ is an exact category, where $\mathcal{A}^{\mathrm{op}}$ is the opposite category of $\mathcal{A}$ and $\mathcal{E}^{\mathrm{op}}$ consists of the pairs $(d, i)$ such that $(i, d)$ is in $\mathcal{E}$.

There is always a unique minimal exact structure $\mathcal{E}_{0}$ on a given additive category $\mathcal{A}$ with splitting idempotents. Namely, $\mathcal{E}_{0}$ consists of all exact pairs $(i, d)$ such that $d$ is a retraction and $i$ is a kernel of $d$ (or alternatively $i$ is a section and $d$ is a cokernel of $i$ ). The exact structure $\mathcal{E}_{0}$ is called the split structure. On the other hand, if $\mathcal{A}$ happens to be abelian, then the collection $\mathcal{E}_{\mathcal{A}}$ of all exact sequences in $\mathcal{A}$ is an exact structure which obviously is the unique maximal exact structure on $\mathcal{A}$.

1.2. Relative theory in exact categories. Let us fix an exact category $(\mathcal{A}, \mathcal{E})$. For given objects $A$ and $C$ of $\mathcal{A}$ we denote by $\operatorname{Ext}_{\mathcal{E}}(C, A)$ the set of all exact pairs $A \stackrel{i}{\rightarrow} B \stackrel{d}{\rightarrow} C$ in $\mathcal{E}$ modulo the equivalence relation which is defined in the following usual way. Two such pairs $(i, d)$ and $\left(i^{\prime}, d^{\prime}\right)$ are equivalent if there exists a commutative diagram as below:

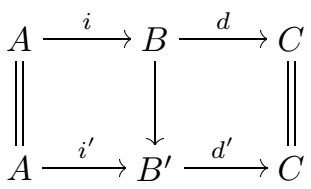

Since any additive category has finite direct sums and in particular uniquely defined diagonal and codiagonal maps, and since an exact category has pullback and pushout pairs, $\operatorname{Ext}_{\mathcal{E}}(C, A)$ becomes an abelian group under Baer sum.

As above, we denote by $\mathcal{A}^{\mathrm{op}}$ the opposite category of $\mathcal{A}$. A functor $F: \mathcal{A}^{\mathrm{op}} \times \mathcal{A} \rightarrow$ $\mathcal{C}$ is called a bifunctor, where $\mathcal{C}$ is some (additive) category. We say that a bifunctor $F$ is additive if the induced functors $F(C$,$) and F(, A)$ are additive for all objects $A$ and $C$ in $\mathcal{A}$. Let $F: \mathcal{A}^{\mathrm{op}} \times \mathcal{A} \rightarrow \mathcal{C}$ be a bifunctor. A subbifunctor $G: \mathcal{A}^{\mathrm{op}} \times \mathcal{A} \rightarrow \mathcal{C}$ of $F$ is said to be additive if $G$ is an additive bifunctor.

We have that $\operatorname{Ext}_{\mathcal{E}}($,$) defines an additive bifunctor \mathcal{A}^{\mathrm{op}} \times \mathcal{A} \rightarrow \mathrm{Ab}$, where $\mathrm{Ab}$ denotes the category of all abelian groups. Let $F$ be a subbifunctor of $\operatorname{Ext}_{\mathcal{E}}($,$) .$ An exact pair

$$
(i, d): A \stackrel{i}{\rightarrow} B \stackrel{d}{\rightarrow} C
$$

is said to be $F$-exact if $(i, d)$ is in $F(C, A)$. Being a subbifunctor in particular means that every pullback and pushout pair of an $F$-exact pair $A \stackrel{i}{\rightarrow} B \stackrel{d}{\rightarrow} C$ given by maps $C^{\prime} \rightarrow C$ and $A \rightarrow A^{\prime}$ is again $F$-exact. In particular, a subbifunctor $F$ of $\operatorname{Ext}_{\mathcal{E}}($,$) determines a collection of exact pairs which is closed under isomorphisms,$ pushout pairs and pullback pairs. Conversely, any nonempty collection of exact pairs which is closed under isomorphisms, pushout and pullback pairs, gives rise to a subbifunctor of $\operatorname{Ext}_{\mathcal{E}}():, \mathcal{A}^{\mathrm{op}} \times \mathcal{A} \rightarrow$ Sets in the obvious way.

As we are interested in additive subbifunctors of $\operatorname{Ext}_{\mathcal{E}}($,$) , the following result$ is useful.

Lemma 1.2. Let $F$ be a subbifunctor of $\operatorname{Ext}_{\mathcal{E}}():, \mathcal{A}^{\mathrm{op}} \times \mathcal{A} \rightarrow$ Sets. Then $F$ is an additive subbifunctor of $\operatorname{Ext}_{\mathcal{E}}():, \mathcal{A}^{\mathrm{op}} \times \mathcal{A} \rightarrow \mathrm{Ab}$ if and only if $F$ is closed under direct sums of F-exact pairs. 
Next we explain the connection between exact structures and relative theories given by subbifunctors of $\operatorname{Ext}_{\mathcal{E}}($,$) . Let F$ be a subbifunctor of $\operatorname{Ext}_{\mathcal{E}}($,$) . Define$ $\mathcal{M}_{F}$ to be the class of morphisms in $\mathcal{A}$ containing all the deflations and inflations of all $F$-exact pairs, and all morphisms whose kernels and cokernels exist and belong to an $F$-exact pair.

Consider the following properties for a class $\mathcal{M}$ of morphisms in $\mathcal{A}$.

A $\mathcal{M}$ contains all zero inflations and deflations in $\mathcal{A}$.

B If $\alpha$ is in $\mathcal{M}$ and $\alpha=\sigma \beta \tau$ for some isomorphisms $\sigma$ and $\tau$, then $\beta$ is in $\mathcal{M}$.

C $\alpha$ is in $\mathcal{M}$ if and only if $\operatorname{Ker} \alpha$ and Coker $\alpha$ exist and are in $\mathcal{M}$.

$\mathrm{D}$ If $\beta(\alpha)$ and $\alpha \beta$ are inflations (deflations) and $\alpha \beta$ is in $\mathcal{M}$, then $\beta(\alpha)$ is in $\mathcal{M}$.

$\mathrm{E}_{1}$ If $\alpha$ and $\beta$ are inflations in $\mathcal{M}$ and $\alpha \beta$ is defined, then $\alpha \beta$ is in $\mathcal{M}$.

$\mathrm{E}_{2}$ If $\alpha$ and $\beta$ are deflations in $\mathcal{M}$ and $\alpha \beta$ is defined, then $\alpha \beta$ is in $\mathcal{M}$.

A class of morphisms satisfying the properties A-D is called an $f$. class in [BH]. Generalizing results from $[\mathrm{BH}]$, we have that the above family $\mathcal{M}_{F}$ of morphisms is an f. class. Note that when $F$ induces an exact structure on $\mathcal{A}$, then $\mathrm{E}_{1}$ and $\mathrm{E}_{2}$ are automatically satisfied for $\mathcal{M}_{F}$, by Proposition 1.1 and the axiom E1 of an exact category. Conversely, again generalizing results from $[\mathrm{BH}]$, one can show that if $\mathcal{M}$ is an $\mathrm{f}$. class of morphisms, then it naturally gives rise to a subbifunctor of $\operatorname{Ext}_{\mathcal{E}}($,$) . Since a subbifunctor F$ of $\operatorname{Ext}_{\mathcal{E}}():, \mathcal{A}^{\mathrm{op}} \times \mathcal{A} \rightarrow$ Sets is additive if and only if the $F$-exact pairs are closed under direct sums, it follows that a class of morphisms $\mathcal{M}$ in $\mathcal{A}$ gives rise to an additive subbifunctor of $\operatorname{Ext}_{\mathcal{E}}($, ) if and only if $\mathcal{M}$ is an f. class and $\mathcal{M}$ is closed under direct sums of deflations, or equivalently closed under direct sums of inflations.

Recall from $[\mathrm{BH}]$ that an additive subbifunctor $F$ of $\operatorname{Ext}_{\mathcal{E}}($,$) is said to be closed$ on the right (left) if $F(A),(F(, A))$ is half exact on $F$-exact pairs for all $A$ in $\mathcal{A}$. The subbifunctor $F$ is said to be closed if it is both left and right closed. Then the following theorem stated for abelian categories in $[\mathrm{BH}]$ is easily generalized to our setting.

Theorem 1.3 ([BH, Theorem 1.1]). Let $F$ be an additive subbifunctor of $\operatorname{Ext}_{\mathcal{E}}(,$,$) .$ Then $F$ is closed on the right (resp. left) if and only if the class $\mathcal{M}_{F}$ satisfies $\mathrm{E}_{1}$ (resp. $\mathrm{E}_{2}$ ).

Let $F$ be an additive subbifunctor of $\operatorname{Ext}_{\mathcal{E}}($,$) . It is natural to ask when F$ induces an exact structure on $\mathcal{A}$. The subbifunctor $F$ defines a class $\mathcal{E}_{F}$ of exact pairs closed under isomorphisms, namely the $F$-exact pairs. Since the $F$-exact pairs are closed under pullbacks and pushouts, the axioms Ex2 and Ex2 $2^{\text {op }}$ are clearly satisfied. Since $F(0,0)$ is a subgroup of $\operatorname{Ext}_{\mathcal{E}}($,$) , it contains the split exact pairs$ starting and ending in the zero object. Hence the identity morphism $i_{0}$ of the zero object occurs as a deflation in an $F$-exact pair. The axiom Ex1 is satisfied if and only if $F$ is closed on the left. Hence we have proved the first equivalence of the following result.

Proposition 1.4. Let $(\mathcal{A}, \mathcal{E})$ be an exact category and $F$ an additive subbifunctor of $\operatorname{Ext}_{\mathcal{E}}():, \mathcal{A}^{\mathrm{op}} \times \mathcal{A} \rightarrow \mathrm{Ab}$. The following assertions are equivalent.

(a) The class $\mathcal{E}_{F}$ is an exact structure on $\mathcal{A}$.

(b) $F$ is closed on the left.

(c) $F$ is closed.

(d) $F$ is closed on the right. 
Proof. The equivalence of (a) and (b) we already saw. Suppose that $F$ is a left closed subbifunctor of $\operatorname{Ext} t_{\mathcal{E}}($,$) and consequently \mathcal{E}_{F}$ is an exact structure on $\mathcal{A}$. By Proposition 1.1 the composition of two inflations in this exact structure is again an inflation. This implies that $F$ is also closed on the right by Theorem 1.3. Therefore (b) implies both (c) and (d). The remaining implications are clear or follow by duality.

Using Theorem 1.3, it is proved for abelian categories in $[\mathrm{BH}]$ that the intersection of closed additive subbifunctors of $\operatorname{Ext}_{\mathcal{E}}($,$) again is closed ([BH, Proposition$ 1.3]). This result generalizes directly to our setting and gives the following using Proposition 1.4.

Corollary 1.5. Let $\left\{F_{i}\right\}_{i \in I}$ be a family of additive subbifunctors of $\operatorname{Ext}_{\mathcal{E}}($,$) . If$ $F_{i}$ is closed for all $i$ in $I$, then the intersection $\bigcap_{i \in I} F_{i}$ is closed.

Another application of the above theorem is the following characterization of exact structures in abelian categories.

Corollary 1.6. If $\mathcal{A}$ is an abelian category, then any exact structure $\mathcal{E}$ on $\mathcal{A}$ is of the shape $\mathcal{E}_{F}$ for some closed additive subbifunctor $F$ of $\operatorname{Ext}_{\mathcal{A}}($,$) .$

Proof. Let $\mathcal{E}$ be any exact structure on $\mathcal{A}$. Then we have seen that the exact pairs in $\mathcal{E}$ are closed under isomorphisms, pullbacks and pushouts. Hence $\mathcal{E}$ defines a closed subbifunctor $F_{\mathcal{E}}$ of $\operatorname{Ext}_{\mathcal{A}}($,$) . It is proved in [BH, Theorem 1.1] that a$ closed subbifunctor of $\operatorname{Ext}_{\mathcal{A}}($,$) is additive. This completes the proof.$

1.3. Basic examples of closed subbifunctors. Let $(\mathcal{A}, \mathcal{E})$ be an exact category and let $\mathcal{X}$ be a full subcategory of $\mathcal{A}$. For each pair of objects $A$ and $C$ in $\mathcal{A}$ define

$$
F_{\mathcal{X}}(C, A)=\{A \rightarrow B \rightarrow C \in \mathcal{E} \mid(\mathcal{X}, B) \rightarrow(\mathcal{X}, C) \rightarrow 0 \text { is exact }\}
$$

and

$$
F^{\mathcal{X}}(C, A)=\{A \rightarrow B \rightarrow C \in \mathcal{E} \mid(B, \mathcal{X}) \rightarrow(A, \mathcal{X}) \rightarrow 0 \text { is exact }\} .
$$

Generalizing a result from [ASo], these constructions give additive subbifunctors of $\operatorname{Ext}_{\mathcal{E}}($,$) . Moreover they are obviously closed.$

Proposition 1.7. The additive subbifunctors $F_{\mathcal{X}}$ and $F^{\mathcal{X}}$ of $\operatorname{Ext}_{\mathcal{E}}($,$) are closed$ for any subcategory $\mathcal{X}$ of $\mathcal{A}$.

A classical situation for this construction is the following. Let $\Lambda$ be an artin algebra and $\Gamma$ be a subalgebra of $\Lambda$. Then it is well-known that the collection of exact sequences in mod $\Lambda$ such that they are split exact as sequences over $\Gamma$, defines a subbifunctor $F$ of $\operatorname{Ext}_{\Lambda}^{1}($,$) which coincides with F_{\Lambda \otimes_{\Gamma} \bmod \Gamma}$.

We have the following inclusions between classes of additive subbifunctors of $\operatorname{Ext}_{\mathcal{E}}(,):, \mathcal{A}^{\mathrm{op}} \times \mathcal{A} \rightarrow \mathrm{Ab}:$

$\left\{F_{\mathcal{X}} \mid \mathcal{X}\right.$ full subcategory of $\left.\mathcal{A}\right\} \subset\{$ closed subbifunctors $\} \subset\{$ all subbifunctors $\}$.

Now let $\mathcal{A}=\bmod \Lambda$ for an artin algebra $\Lambda$. Let $F$ be the additive subbifunctor generated by all almost split sequences in $\bmod \Lambda$. It is easy to see that an exact sequence $\eta$ is $F$-exact if and only if $\eta$ is a direct sum of split exact and almost split exact sequences. Suppose $\bmod \Lambda$ has an almost split sequence with a nonprojective middle term $0 \rightarrow A \rightarrow B \rightarrow C \rightarrow 0$. Let $0 \rightarrow D$ Tr $B^{\prime} \rightarrow B^{\prime \prime} \rightarrow B^{\prime} \rightarrow 0$ be the almost split sequence ending in a non-projective indecomposable summand $B^{\prime}$ of $B$. Then the end terms of the complex $F\left(C, D \operatorname{Tr} B^{\prime}\right) \rightarrow F\left(B, D \operatorname{Tr} B^{\prime}\right) \rightarrow$ 
$F\left(A, D \operatorname{Tr} B^{\prime}\right)$ are zero while the middle term is non-zero. Therefore $F$ is not closed. This shows that the last inclusion is proper.

Next we observe that for artin algebras $\Lambda$ of finite type all closed subbifunctors $F$ of $\operatorname{Ext}_{\Lambda}^{1}($,$) are of the form F_{\mathcal{X}}$ for some subcategory of $\bmod \Lambda$. Denote by $\mathcal{P}(F)$ the full additive subcategory of $\bmod \Lambda$ consisting of the modules $P$ such that $\operatorname{Hom}_{\Lambda}(P$,$) is exact on all F$-exact sequences.

Proposition 1.8. Let $\Lambda$ be an artin algebra of finite type. If $F$ is a closed additive subbifunctor of $\operatorname{Ext}_{\Lambda}^{1}($,$) , then F=F_{\mathcal{P}(F)}$.

Proof. Suppose $\Lambda$ is an artin algebra of finite type and that $F$ is a closed additive subbifunctor of $\operatorname{Ext}_{\Lambda}^{1}($,$) . We want to show that F=F_{\mathcal{P}(F)}$. Since $F \subset F_{\mathcal{P}(F)}$, it is enough to show that an $F_{\mathcal{P}(F)}$-exact sequence of the form $0 \rightarrow K \rightarrow P \rightarrow C \rightarrow 0$ with $P$ in $\mathcal{P}(F)$ is $F$-exact, where $C$ is any module in $\bmod \Lambda$ which is not in $\mathcal{P}(F)$.

Let $C$ be in $\bmod \Lambda$ but not in $\mathcal{P}(F)$. Let $f_{1}: P_{1} \oplus B_{1} \rightarrow C \rightarrow 0$ be the minimal right almost split map ending in $C$, where $P_{1}$ is in $\mathcal{P}(F)$ and $B_{1}$ does not have any nonzero summands from $\mathcal{P}(F)$. Then $f_{1}$ is a deflation in the exact structure $\mathcal{E}_{F}$ on $\bmod \Lambda$ defined by $F$. Let $f_{2}: P_{2} \oplus B_{2} \rightarrow B_{1}$ be the direct sum of the minimal right almost split maps ending in the different indecomposable direct summands of $B_{1}$, where $P_{2}$ is in $\mathcal{P}(F)$ and $B_{2}$ does not have any nonzero summands from $\mathcal{P}(F)$. Then $f_{1}\left(\operatorname{id}_{P_{1}}, f_{2}\right)=f_{1} f_{2}^{\prime}$ is a deflation in $\mathcal{E}_{F}$. We can continue this procedure and get a composition of deflations

$$
f_{1} f_{2}^{\prime} f_{3}^{\prime} \cdots f_{n}^{\prime}: P_{1} \oplus P_{2} \oplus \cdots \oplus P_{n} \oplus B_{n} \rightarrow C,
$$

which is in $\mathcal{E}_{F}$. If $B_{n}$ is zero for some $n$, then our claim follows. If $B_{n}$ is never zero, then the induced morphism from $B_{n}$ to $C$ must be zero for some $n$ due to the Harada-Sai lemma. Using this $n$ and considering the $F$-exact sequence it induces, the $B_{n}$-part will split off and we get our desired sequence. This completes the proof of the proposition.

1.4. Construction of subbifunctors of $\operatorname{Ext}_{\mathcal{E}}(, \quad)$ from other exact categories. We saw above that for any subcategory $\mathcal{X}$ of $\mathcal{A}$ the subbifunctors $F_{\mathcal{X}}$ and $F^{\mathcal{X}}$ are closed subbifunctors of $\operatorname{Ext}_{\mathcal{E}}($,$) , and therefore induce an exact structure$ on $\mathcal{A}$. In this section we discuss another method for constructing additive subbifunctors of $\operatorname{Ext}_{\mathcal{E}}($,$) for an exact category \mathcal{A}$, which again gives rise to an exact structure on $\mathcal{A}$.

Let $(\mathcal{A}, \mathcal{E})$ and $\left(\mathcal{A}^{\prime}, \mathcal{E}^{\prime}\right)$ be two exact categories and let $G: \mathcal{A} \rightarrow \mathcal{A}^{\prime}$ be an additive functor. Let $F_{0}$ be an additive subbifunctor of $\operatorname{Ext}_{\mathcal{E}^{\prime}}($,$) . Define for each pair A$ and $C$ of objects in $\mathcal{A}$

$$
F_{\left[G, F_{0}\right]}(C, A)=\left\{A \rightarrow B \rightarrow C \in \mathcal{E} \mid G(A) \rightarrow G(B) \rightarrow G(C) \text { is } F_{0} \text {-exact }\right\} .
$$

This does not necessarily define an additive subbifunctor of $\operatorname{Ext}_{\mathcal{E}}($,$) in general. It$ is easy to see the following.

Lemma 1.9. The subsets $F_{\left[G, F_{0}\right]}(C, A)$ for all objects $A$ and $C$ of $\mathcal{A}$ define an additive subbifunctor of $\operatorname{Ext}_{\mathcal{E}}():, \mathcal{A}^{\mathrm{op}} \times \mathcal{A} \rightarrow \mathrm{Ab}$ if and only if the functor $G$ has the following properties.

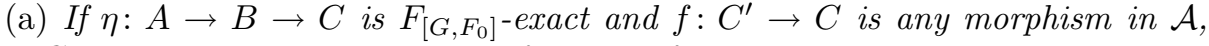
then $G$ applied to the pullback pair of $\eta$ along $f$ is exact.

(b) If $\eta: A \rightarrow B \rightarrow C$ is $F_{\left[G, F_{0}\right]}$-exact and $g: A \rightarrow A^{\prime}$ is any morphism in $\mathcal{A}$, then $G$ applied to the pushout pair of $\eta$ along $g$ is exact. 
We are interested in when $F_{\left[G, F_{0}\right]}$ induces an exact structure on $\mathcal{A}$. A sufficient condition for this is given in the next result.

Proposition 1.10. Assume that $F_{\left[G, F_{0}\right]}$ is an additive subbifunctor of $\operatorname{Ext}_{\mathcal{E}}($,$) .$ If $F_{0}$ is closed, then $F_{\left[G, F_{0}\right]}$ is closed.

Proof. Assume that $F_{0}$ is closed. Assume that $A \stackrel{\alpha}{\rightarrow} B \stackrel{\alpha^{\prime}}{\rightarrow} C$ and $A^{\prime} \stackrel{\beta}{\rightarrow} A \stackrel{\beta^{\prime}}{\rightarrow} C^{\prime}$ are $F_{\left[G, F_{0}\right]}$-exact. It is proved in $[\mathrm{Ke} 2]$ that we have the following exact commutative diagram:

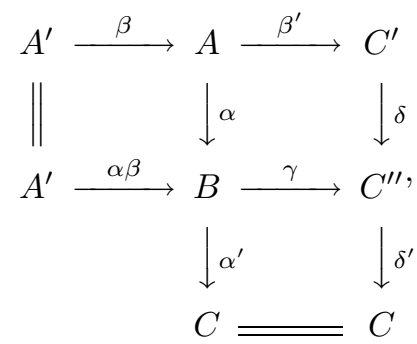

where all rows and columns are exact pairs and the upper right hand square is a pushout square. The last fact implies that the last column is $F_{\left[G, F_{0}\right]}$-exact. Applying the functor $G$ we obtain the commutative diagram

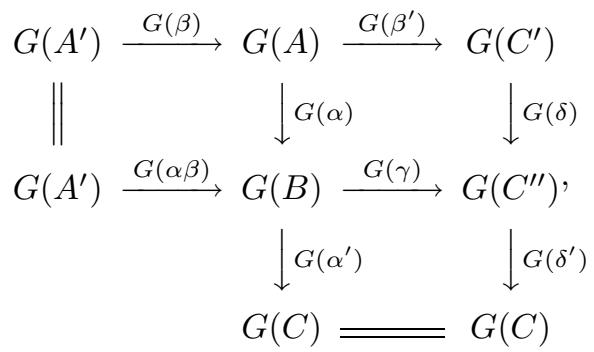

where the upper row and the columns are $F_{0}$-exact.

Assume that $t: X \rightarrow G(B)$ is such that $G(\gamma) t=0$. Then $G\left(\delta^{\prime}\right) G(\gamma) t=G\left(\alpha^{\prime}\right) t=$ 0 . Since the middle column is $F_{0}$-exact, there exists a unique $t^{\prime}: X \rightarrow G(A)$ such that $G(\alpha) t^{\prime}=t$. We have that $0=G(\gamma) G(\alpha) t^{\prime}=G(\delta) G\left(\beta^{\prime}\right) t^{\prime}$. Therefore $G\left(\beta^{\prime}\right) t^{\prime}=0$, since the last column is $F_{0}$-exact. Now, since the upper row is $F_{0^{-}}$ exact, there exists a unique $t^{\prime \prime}: X \rightarrow G\left(A^{\prime}\right)$ such that $G(\beta) t^{\prime \prime}=t^{\prime}$ and consequently $G(\alpha) G(\beta) t^{\prime \prime}=G(\alpha) t^{\prime}=t$. This shows that $G(\alpha \beta)$ is the kernel of $G(\gamma)$.

Assume that $s: G(B) \rightarrow X$ is such that $s G(\alpha \beta)=0$. Then $(s G(\alpha)) G(\beta)=0$, so that there exists a unique $s^{\prime}: G\left(C^{\prime}\right) \rightarrow X$ such that $s^{\prime} G\left(\beta^{\prime}\right)=s G(\alpha)$. By the same arguments from $[\mathrm{Ke} 2]$ as above, the upper right hand square of the above diagram is a pushout square. This gives the existence of a unique $s^{\prime \prime}: G\left(C^{\prime \prime}\right) \rightarrow X$ such that $s^{\prime \prime} G(\gamma)=s$. This shows that $G(\gamma)$ is the cokernel of $G(\alpha \beta)$. Hence it follows that the middle row $\eta$ is an exact pair. Since $F_{0}$ is closed, $\eta$ is $F_{0}$-exact and therefore $A^{\prime} \stackrel{\alpha \beta}{\rightarrow} B \rightarrow C$ is $F_{\left[G, F_{0}\right]}$-exact. This show that $F_{\left[G, F_{0}\right]}$ is closed.

1.5. Exact categories modulo relations. Let $(\mathcal{A}, \mathcal{E})$ be an exact category, and let $\mathfrak{R}$ be a relation on $\mathcal{A}$. This means that for each pair of objects $A$ and $B$ in $\mathcal{A}$ we have a subgroup $\mathfrak{R}(A, B)$ of $\operatorname{Hom}_{\mathcal{A}}(A, B)=\mathcal{A}(A, B)$ such that if $f$ is in $\mathfrak{R}(A, B)$, $g$ in $\operatorname{Hom}_{\mathcal{A}}(B, C)$ and $h$ in $\operatorname{Hom}_{\mathcal{A}}(U, A)$, then $g f$ is in $\mathfrak{R}(A, C)$ and $f h$ in $\mathfrak{R}(U, B)$. 
We denote by $\mathcal{A} / \mathfrak{R}$ the factor category whose objects are the same as those of $\mathcal{A}$, and where the morphisms are given by

$$
\operatorname{Hom}_{\mathcal{A} / \mathfrak{R}}(A, B)=\operatorname{Hom}_{\mathcal{A}}(A, B) / \mathfrak{R}(A, B) .
$$

If $\mathcal{E}$ is an exact structure for $\mathcal{A}$, then we define $\mathcal{E} / \mathfrak{R}$ as the collection of all pairs $(\underline{i}, \underline{d})$ of morphisms in $\mathcal{A} / \mathfrak{R}$ such that $(i, d) \in \mathcal{E}$. We want to give sufficient conditions on $\mathfrak{R}$ for $\mathcal{E} / \mathfrak{R}$ to be an exact structure for $\mathcal{A} / \mathfrak{R}$.

Proposition 1.11. Let $(\mathcal{A}, \mathcal{E})$ be an exact category and $\mathfrak{R}$ a relation on $\mathcal{A}$. Then $(\mathcal{A} / \mathfrak{R}, \mathcal{E} / \mathfrak{R})$ is an exact category provided that the following properties are satisfied.

(I) For all objects $A$ of $\mathcal{A}$ the functors $\mathfrak{R}(A, \quad)$ and $\mathfrak{R}(, A)$ map the exact pairs in $\mathcal{E}$ to short exact sequences of abelian groups.

(II) The relation $\mathfrak{R}$ is contained in the Jacobson radical of $\mathcal{A}$ (i.e. for all objects $A$ of $\mathcal{A}$ and all morphisms $r \in \mathfrak{R}(A, A)$ the map $\operatorname{id}_{A}+r$ is an isomorphism).

Proof. Let us first reformulate condition (I) into the following conditions (i) to (iv):

(i) If $t d$ is in $\mathfrak{R}$ where $d$ is a deflation, then $t$ is in $\mathfrak{R}$.

(ii) If $i s$ is in $\mathfrak{R}$ where $i$ is an inflation, then $s$ is in $\mathfrak{R}$.

(iii) If $d: B \rightarrow C$ is a deflation and $t: X \rightarrow C$ is in $\mathfrak{R}$, then there exists a morphism $s: X \rightarrow B$ with $d s=t$ and $s$ in $\mathfrak{R}$.

(iv) If $i: A \rightarrow C$ is an inflation and $u: A \rightarrow Y$ is in $\mathfrak{R}$, then there exists a morphism $v: C \rightarrow Y$ with $v i=u$ and $v$ is in $\mathfrak{R}$.

We now use these four conditions for the proof. Let $(i, d)$ be an exact pair in $\mathcal{A}$; that is, we have $A \stackrel{i}{\rightarrow} B \stackrel{d}{\rightarrow} C$ in $\mathcal{E}$. It follows from (i) that $\underline{d}$ in $\mathcal{A} / \mathfrak{R}$ is an epimorphism in $\mathcal{A} / \mathfrak{R}$, and it follows from (ii) that $\underline{i}$ is a monomorphism.

Let $t^{\prime}: X \rightarrow B$ be such that $d t^{\prime}$ is in $\Re$. Then by (iii) there is some morphism $s: X \rightarrow B$ with $s$ in $\mathfrak{R}$ and $d s=d t^{\prime}$; that is, $d\left(s-t^{\prime}\right)=0$. Since $i: A \rightarrow B$ is a kernel for $d: B \rightarrow C$, there is then a map $a: X \rightarrow A$ with $i a=-s+t^{\prime}$ where $s$ is in $\mathfrak{R}$, so $i a+\mathfrak{R}=t^{\prime}+\mathfrak{R}$. This shows that $\underline{i}: A \rightarrow B$ is a kernel of $\underline{d}: B \rightarrow C$ in $\mathcal{A} / \mathfrak{R}$.

Similarly we prove that $\underline{d}: B \rightarrow C$ is a cokernel of $\underline{i}: A \rightarrow B$.

Since the deflations in $\mathcal{A} / \mathfrak{R}$ are those induced from $\mathcal{A}$, it follows directly from the fact that $\mathcal{A}$ is an exact category that $\mathcal{E} / \mathfrak{R}$ satisfies the property E1. The same direct argument shows that $\mathcal{E} / \mathfrak{R}$ has property E2. For properties E3 and E3 ${ }^{\text {op }}$ we have to use property (II).

Assume that $\underline{d e}$ is a deflation in $\mathcal{A} / \mathfrak{R}$; that is, $\underline{d e}=\underline{u}$ for a deflation $u$ in $\mathcal{A}$. Hence $d e=u+r$ for some liftings $d$ and $e$ of $\underline{d}$ and $\underline{e}$ and some element $r$ in $\mathfrak{R}$. Since $u: B \rightarrow C$ is a deflation and $r: B \rightarrow C$ is in $\Re$, there exists a map $s: B \rightarrow B$ in $\mathfrak{R}$ such that $r=u s$ by (iii). Hence $d e=u\left(\operatorname{id}_{B}+s\right)$ for some element $s$ in $\mathfrak{R}$. By property (II) $\mathrm{id}_{B}+s$ is a deflation, so that $u\left(\mathrm{id}_{B}+s\right)=d e$ is a deflation. Now, since $\mathcal{A}$ is an exact category it follows that $d$ is a deflation, and therefore $\underline{d}$ is a deflation in $\mathcal{A} / \mathfrak{R}$.

Similarly we prove that if $\underline{j} \underline{i}$ is an inflation in $\mathcal{A} / \mathfrak{R}$, then $\underline{i}$ is an inflation in $\mathcal{A} / \mathfrak{R}$.

Identities are deflations and inflations in $\mathcal{A}$, and therefore they also are deflations and inflations in $\mathcal{A} / \mathfrak{R}$. This completes the proof of the proposition. 


\section{EXACT CATEGORIES WITH ALMOST SPLIT PAIRS}

2.1. Projective and injective objects. Suppose $(\mathcal{A}, \mathcal{E})$ is an exact category. An object $P$ of $\mathcal{A}$ is called $\mathcal{E}$-projective if each exact pair $X \stackrel{i}{\rightarrow} Y \stackrel{d}{\rightarrow} P$ in $\mathcal{E}$ splits (i.e. lies in $\mathcal{E}_{0}$ ). The $\mathcal{E}$-injective objects are defined dually. If the underlying exact structure $\mathcal{E}$ is clear, we speak only of projective and injective objects.

Using the following lemma, one sees that the $\mathcal{E}$-projective objects are precisely those objects $P$ such that for each pair $X \stackrel{i}{\rightarrow} Y \stackrel{d}{\rightarrow} Z$ in $\mathcal{E}$ the sequence

$$
0 \rightarrow \mathcal{A}(P, X) \longrightarrow \mathcal{A}(P, Y) \longrightarrow \mathcal{A}(P, Z) \rightarrow 0
$$

obtained by applying the functor $\mathcal{A}(P$,$) is a short exact sequence of abelian groups.$ Of course, $\mathcal{E}$-injectives are characterized in the dual fashion.

Lemma 2.1. Suppose that in an exact category $(\mathcal{A}, \mathcal{E})$ there is a commutative diagram

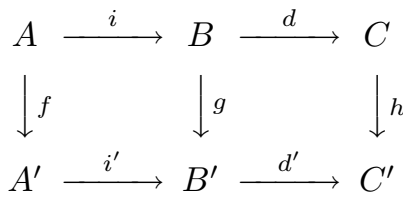

such that $(i, d)$ and $\left(i^{\prime}, d^{\prime}\right)$ are exact pairs in $\mathcal{E}$. Then there exists a morphism $s: B \rightarrow A^{\prime}$ such that si $=f$ if and only if there exists a morphism $t: C \rightarrow B^{\prime}$ such that $d^{\prime} t=h$.

We denote by $\mathcal{P}(\mathcal{E})$ and $\mathcal{I}(\mathcal{E})$ the full categories of $\mathcal{A}$ formed by the projective and injective objects. The subcategories $\mathcal{P}(\mathcal{E})$ and $\mathcal{I}(\mathcal{E})$ are closed under finite direct sums and direct summands.

2.2. Almost split maps and pairs. From now on we restrict ourselves to KrullSchmidt categories, that is, additive categories where each object is a finite direct sum of indecomposable objects with local endomorphism rings. Thus the indecomposable objects coincide with those having local endomorphism rings.

A morphism $g: Y \rightarrow Z$ in a Krull-Schmidt category $\mathcal{A}$ is called right almost split if it is not a retraction and for any non-retraction $t: A \rightarrow Z$ there exists a morphism $s: A \rightarrow Y$ such that $t=g s$. Given a right almost split map $g: Y \rightarrow Z$, the set $g \mathcal{A}(Z, Y)$ is the unique maximal proper right ideal of the $\operatorname{ring} \mathcal{A}(Z, Z)$ and consequently $Z$ has a local endomorphism ring. In particular, $Z$ has to be indecomposable. We say that $\mathcal{A}$ has right almost split morphisms if for all indecomposable objects $Z$ there exists a right almost split morphism ending in $Z$. Dually we define left almost split morphisms. We say that $\mathcal{A}$ has almost split morphisms if $\mathcal{A}$ has right and left almost split morphisms.

A morphism $g: Y \rightarrow Z$ is called right minimal if every endomorphism $s: Y \rightarrow Y$ with the property that $g=g s$, is an isomorphism. Minimal right almost split morphisms ending in an object $Z$ are essentially unique. Namely, if $g: Y \rightarrow Z$ and $g^{\prime}: Y^{\prime} \rightarrow Z$ are right minimal almost split morphisms, then there is an isomorphism $s: Y^{\prime} \rightarrow Y$ satisfying $g^{\prime}=g s$. If right almost split morphisms exist, it is not clear a priori that minimal right almost split morphisms will exist. We show next that this is the case in our setting. 
Proposition 2.2. Let $\mathcal{A}$ be a Krull-Schmidt category and $Z$ an object of $\mathcal{A}$ in which a right almost split map ends. Then there exists a minimal right almost split map $Y \rightarrow Z$.

Proof. It is easy to see that one obtains a minimal right almost split map by choosing a right almost split map $Y \rightarrow Z$ in $\mathcal{A}$ such that $Y$ is a direct sum of indecomposable objects with the smallest possible number of summands.

Suppose now that the Krull-Schmidt category $\mathcal{A}$ carries an exact structure $\mathcal{E}$. Using Lemma 2.1, the proof of the following proposition coincides with the usual one for module categories.

Proposition 2.3. Suppose $(\mathcal{A}, \mathcal{E})$ is an exact category such that $\mathcal{A}$ is Krull-Schmidt and $X \stackrel{f}{\rightarrow} Y \stackrel{g}{\rightarrow} Z$ is an exact pair in $\mathcal{E}$. Then the following assertions are equivalent.

(i) $f$ is minimal left almost split.

(ii) $g$ is minimal right almost split.

(iii) $f$ is left almost split and $g$ is right almost split.

An exact pair $X \stackrel{f}{\rightarrow} Y \stackrel{g}{\rightarrow} Z$ in $\mathcal{E}$ as in the above proposition is said to be an almost split pair. Clearly such an almost split pair can only exist provided $X$ is indecomposable non- $\mathcal{E}$-injective and $Z$ is indecomposable non- $\mathcal{E}$-projective. The exact category $(\mathcal{A}, \mathcal{E})$ is said to have almost split pairs if $\mathcal{A}$ has almost split morphisms and moreover for all indecomposable non-projective objects $Z$ there exists an almost split pair $X \stackrel{f}{\rightarrow} Y \stackrel{g}{\rightarrow} Z$ and dually for all indecomposable noninjective objects $X$ there exists an almost split pair $X \stackrel{f}{\rightarrow} Y \stackrel{g}{\rightarrow} Z$.

The uniqueness of minimal almost split maps shows that almost split pairs $X \stackrel{f}{\rightarrow}$ $Y \stackrel{g}{\rightarrow} Z$ are uniquely determined by $X$ or $Z$. We introduce the notation $X=\tau Z$ (and $Z=\tau^{-1} X$ ) whenever $X \stackrel{f}{\rightarrow} Y \stackrel{g}{\rightarrow} Z$ is an almost split pair.

Of course the main examples of exact categories having almost split sequences are the categories of finitely generated modules over an artin algebra $\Lambda$, where $\tau$ coincides with $D$ Tr. One obtains trivial examples by considering Krull-Schmidt categories $\mathcal{A}$ equipped with the split structure $\mathcal{E}_{0}$. For this structure each object is projective and injective. Hence $\left(\mathcal{A}, \mathcal{E}_{0}\right)$ has almost split pairs provided $\mathcal{A}$ has almost split morphisms (which is always the case if $\mathcal{A}$ has only finitely many indecomposable objects up to isomorphism). Further examples of exact categories with almost split pairs appearing as subcategories of module categories can be found in $[\mathrm{Au}]$ and $[\mathrm{ASm}]$.

The following result is well-known for module categories over artin algebras [Au] (see also [ARS, Ch. IV]).

Proposition 2.4. Let $(\mathcal{A}, \mathcal{E})$ be an exact category with almost split pairs. Then for an exact pair $A \stackrel{f}{\rightarrow} B \stackrel{g}{\rightarrow} C$ in $\mathcal{E}$ and an indecomposable non-projective object $X$ in $\mathcal{A}$ the following are equivalent.

(a) Every map $X \rightarrow C$ factors through $g: B \rightarrow C$.

(b) Every map $A \rightarrow \tau X$ factors through $f: A \rightarrow B$.

Proof. Assume first that every map $X \rightarrow C$ factors through $g: B \rightarrow C$. Assume to the contrary that there is some map $h: A \rightarrow \tau X$ which does not factor through 
$f: A \rightarrow B$. Taking the pushout we have the following exact commutative diagram:

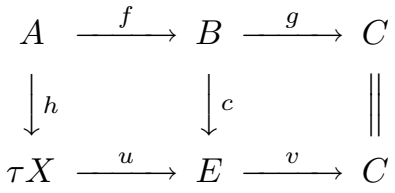

with the lower row non-split.

Let $\tau X \stackrel{\alpha}{\rightarrow} Y \rightarrow X$ be an almost split pair. Since $u: \tau X \rightarrow E$ is not a section, we have an exact commutative diagram

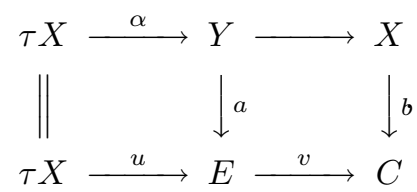

By our assumption that the map $b: X \rightarrow C$ factors through $g: B \rightarrow C$, it follows that is there is a map $t: X \rightarrow B$ such that $b=g t$. Therefore $b=v c t=v t^{\prime}$ for $t^{\prime}=c t: X \rightarrow E$. By Lemma 2.1 there is a map $s: Y \rightarrow \tau X$ such that $s \alpha=\operatorname{id}_{\tau X}$, so that the almost split pair splits, a contradiction.

The dual argument completes the proof.

We put $\tau P=0$ for all indecomposable projective objects of $\mathcal{A}$, and dually $\tau^{-} I=0$ for all indecomposable injective objects. Finally, we extend $\tau$ and $\tau^{-}$to all objects of $\mathcal{A}$ in the obvious way. Then the previous proposition has the following immediate consequence, which is a generalization of a result from [ASo].

Corollary 2.5. If $\mathcal{X}$ is any full subcategory of $\mathcal{A}$, then $F_{\mathcal{X}}=F^{\tau \mathcal{X}}$.

2.3. Injective and projective objects for relative theories. Let $(\mathcal{A}, \mathcal{E})$ be an exact category and let $F$ be an additive subbifunctor of $\operatorname{Ext}_{\mathcal{E}}():, \mathcal{A}^{\mathrm{op}} \times \mathcal{A} \rightarrow \mathrm{Ab}$. With $F$ we associate the full subcategory $\mathcal{P}(F)$ of $\mathcal{A}$ consisting of all objects $P$ such that if $A \rightarrow B \rightarrow C$ is $F$-exact, the sequence $0 \rightarrow(P, A) \rightarrow(P, B) \rightarrow(P, C) \rightarrow 0$ is exact. This subcategory is called the subcategory of $F$-projective objects (see [ASo]). Dually we define the full subcategory $\mathcal{I}(F)$ of $F$-injective objects.

For two subbifunctors $F_{1} \subseteq F_{2}$ we have $\mathcal{P}\left(F_{1}\right) \supseteq \mathcal{P}\left(F_{2}\right)$ and $\mathcal{I}\left(F_{1}\right) \supseteq \mathcal{I}\left(F_{2}\right)$. In particular, $\mathcal{P}(\mathcal{E})=\mathcal{P}\left(\operatorname{Ext}_{\mathcal{E}}(),\right)$ is contained in $\mathcal{P}(F)$ and $\mathcal{I}(\mathcal{E})=\mathcal{I}\left(\operatorname{Ext}_{\mathcal{E}}(),\right)$ is contained in $\mathcal{I}(F)$ for any $F$.

Using Proposition 2.4 we prove that the $F$-projective and $F$-injective objects are closely related in an exact category with almost split pairs.

Proposition 2.6. Let $(\mathcal{A}, \mathcal{E})$ be an exact category with almost split pairs and $F$ an additive subbifunctor of $\operatorname{Ext}_{\mathcal{E}}($,$) .$

(a) The subcategory $\mathcal{I}(F)$ is equal to $\tau(\mathcal{P}(F)) \cup \mathcal{I}(\mathcal{E})$.

(b) The subcategory $\mathcal{P}(F)$ is equal to $\tau^{-}(\mathcal{I}(F)) \cup \mathcal{P}(\mathcal{E})$.

Proof. It follows directly from the Proposition 2.4 that $\tau \mathcal{P}(F)$ is contained in $\mathcal{I}(F)$ and that $\tau^{-1}(\mathcal{I}(F))$ is contained in $\mathcal{P}(F)$.

Let $X$ be indecomposable and in $\mathcal{I}(F)$ but not in $\mathcal{I}(\mathcal{E})$. Then again by Proposition 2.4 we get that $X^{\prime}=\tau^{-} X$ is in $\mathcal{P}(F)$ and $X=\tau\left(X^{\prime}\right)$.

The following characterization of the $F$-projective and $F$-injective objects for a subbifunctor $F$ in terms of almost split pairs is useful. The proof easily generalizes from $[\mathrm{ASo}]$. 
Proposition 2.7. Let $(\mathcal{A}, \mathcal{E})$ be an exact category with almost split pairs and $F$ an additive subbifunctor of $\operatorname{Ext}_{\mathcal{E}}($,$) .$

(a) An indecomposable non-projective object $P$ is in $\mathcal{P}(F)$ if and only if the almost split pair $\tau P \rightarrow E \rightarrow P$ is not $F$-exact.

(b) An indecomposable non-injective object $I$ is in $\mathcal{I}(F)$ if and only if the almost split pair $I \rightarrow E \rightarrow \tau^{-} I$ is not $F$-exact.

2.4. Almost split pairs under relative theories and modulo relations. Let us return to the case that $F$ is a closed subbifunctor of the bifunctor $\operatorname{Ext}_{\mathcal{E}}($,$) for$ a given exact category $(\mathcal{A}, \mathcal{E})$. We saw in section 1 that such a subbifunctor yields a new exact structure $\mathcal{E}_{F}$ on $\mathcal{A}$ which is contained in $\mathcal{E}$. From the discussion of the projective and injective objects in $\mathcal{E}_{F}$ at the beginning of this section it follows that $\mathcal{P}\left(\mathcal{E}_{F}\right)=\mathcal{P}(F)$ and $\mathcal{I}\left(\mathcal{E}_{F}\right)=\mathcal{I}(F)$.

Using Proposition 2.7 we get the following result.

Proposition 2.8. Let $(\mathcal{A}, \mathcal{E})$ be an exact category and $F$ a closed additive subbifunctor of $\operatorname{Ext}_{\mathcal{E}}($,$) . If (\mathcal{A}, \mathcal{E})$ has almost split pairs, then the exact category $\left(\mathcal{A}, \mathcal{E}_{F}\right)$ has almost split pairs. These are the almost split pairs in $\mathcal{E}$ which do not end in an $F$-projective object or equivalently do not start in an $F$-injective object.

Proof. Since $F$ is closed, $F$ induces an exact structure on $\mathcal{A}$ by Proposition 1.4. The claim now follows directly from Proposition 2.7.

We end this section with the following observation.

Proposition 2.9. Let $(\mathcal{A}, \mathcal{E})$ be an exact category and let $\mathfrak{R}$ be a relation on $\mathcal{A}$ satisfying conditions (I) and (II) in Proposition 1.11.

(a) Then $\mathcal{P}(\mathcal{E} / \mathfrak{R})=\mathcal{P}(\mathcal{E})$ and $\mathcal{I}(\mathcal{E} / \mathfrak{R})=\mathcal{I}(\mathcal{E})$.

(b) A morphism $f: B \rightarrow C$ is right (left) almost split in $\mathcal{A}$ if and only if $\underline{f}: B \rightarrow$ $C$ is right (left) almost split in $\mathcal{A} / \mathfrak{R}$.

(c) An exact pair $(f, g)$ is an almost split pair in $\mathcal{A}$ if and only if $(\underline{f}, \underline{g})$ is an almost split pair in $\mathcal{A} / \mathfrak{R}$.

(d) $\mathcal{A}$ has almost split morphisms (almost split pairs) if and only if $\mathcal{A} / \mathfrak{R}$ does.

Proof. It follows from Proposition 1.11 that $\mathcal{E} / \mathfrak{R}$ is an exact structure on $\mathcal{A} / \mathfrak{R}$. Further we only have to observe that it follows from (II) that a morphism $h$ in $\mathcal{A}$ is an isomorphism if and only if $\underline{h}$ is an isomorphism in $\mathcal{A} / \mathfrak{R}$, so that $h$ is a retraction in $\mathcal{A}$ if and only if $\underline{h}$ is a retraction in $\mathcal{A} / \mathfrak{R}$.

\section{EXAMPLES}

The main objective in this section is to illustrate the constructions and the results of the previous sections by concrete examples. The examples, or more correctly the family of examples, we consider are all given over an artin algebra $\Lambda$ in terms of an ideal $\underline{\mathrm{a}}$ in $\Lambda$. We write $\operatorname{Hom}_{\Lambda}(\underline{\mathrm{a}})=$,$G , which is a left exact additive$ subfunctor of the identity $\operatorname{id}_{\bmod \Lambda}: \bmod \Lambda \rightarrow \bmod \Lambda$. Denote by $G^{\prime}=\operatorname{id}_{\bmod \Lambda} / G$ the associated quotient functor. Then we study the subbifunctors $F_{[G, 0]}, F_{\left[G^{\prime}, 0\right]}$ and their intersection, and we show that they induce new exact structures on $\bmod \Lambda$ having almost split pairs. Moreover, the relation on $\bmod \Lambda$ given by $\mathfrak{R}=\{f: A \rightarrow$ $\left.B \mid G(f)=0=G^{\prime}(f)\right\}$ with the exact structure on $\bmod \Lambda$ given by the intersection $F_{[G, 0]} \cap F_{\left[G^{\prime}, 0\right]}$ induce an exact structure on $\bmod \Lambda / \mathfrak{R}$ having almost split pairs. We also study the injectives and projectives of the above subbifunctors of $\operatorname{Ext}_{\Lambda}^{1}($, and investigate when they have enough injectives or projectives. 
Throughout this section let $\Lambda$ be an artin algebra and a an ideal in $\Lambda$.

3.1. Examples of the constructions in section 1. Let $G=\operatorname{Hom}_{\Lambda}(\Lambda / \underline{a}, \quad)$ : $\bmod \Lambda \rightarrow \bmod \Lambda$ and $G^{\prime}$ be as above. Denote by $i_{X}: G(X) \rightarrow X$ the natural inclusion for a $\Lambda$-module $X$ in $\bmod \Lambda$.

Let $F_{0}$ be a closed additive subbifunctor of $\operatorname{Ext}_{\Lambda}^{1}($,$) . We want to show that$ $F_{\left[G, F_{0}\right]}$ and $F_{\left[G^{\prime}, F_{0}\right]}$ define additive subbifunctors of $\operatorname{Ext}_{\Lambda}^{1}(, \quad)$ which induce exact structures on $\bmod \Lambda$. This is based on the following lemmas.

Lemma 3.1. Let $0 \rightarrow A \rightarrow B \rightarrow C \rightarrow 0$ be an exact sequence. Then

$$
0 \rightarrow G(A) \rightarrow G(B) \rightarrow G(C) \rightarrow 0
$$

is exact if and only if

$$
0 \rightarrow G^{\prime}(A) \rightarrow G^{\prime}(B) \rightarrow G^{\prime}(C) \rightarrow 0
$$

is exact.

Proof. This observation follows immediately from the Snake Lemma.

Lemma 3.2. Let $\eta: 0 \rightarrow A \rightarrow B \rightarrow C \rightarrow 0$ be an exact sequence such that $G(\eta)$ $\left(G^{\prime}(\eta)\right)$ is $F_{0}$-exact.

(a) Let $\alpha$ : $C^{\prime} \rightarrow C$ be a morphism in $\bmod \Lambda$ and let $\eta^{\prime}: 0 \rightarrow A \rightarrow B^{\prime} \rightarrow C^{\prime} \rightarrow 0$ be the pullback sequence of $\eta$ along the morphism $\alpha$. Then $G\left(\eta^{\prime}\right)\left(G^{\prime}\left(\eta^{\prime}\right)\right)$ is $F_{0}$ exact.

(b) Let $\alpha: A \rightarrow A^{\prime}$ be a morphism in $\bmod \Lambda$ and let $\eta^{\prime}: 0 \rightarrow A^{\prime} \rightarrow B^{\prime} \rightarrow C \rightarrow 0$ be the pushout sequence of $\eta$ along the morphism $\alpha$. Then $G\left(\eta^{\prime}\right)\left(G^{\prime}\left(\eta^{\prime}\right)\right)$ is $F_{0}$-exact.

Proof. (a) Let $\eta: 0 \rightarrow A \rightarrow B \rightarrow C \rightarrow 0$ be an exact sequence such that $G(\eta): 0 \rightarrow$ $G(A) \rightarrow G(B) \rightarrow G(C) \rightarrow 0$ is $F_{0}$-exact. Let $\alpha: C^{\prime} \rightarrow C$ be a morphism in $\bmod \Lambda$. Define $\eta^{\prime}$ to be the pullback of $\eta$ by the morphism $\alpha$. We want to show that $G\left(\eta^{\prime}\right)$ is exact.

Since $G$ is left exact, $i_{C} G(\alpha)=\alpha i_{C^{\prime}}$ and the pullback $\operatorname{Ext}_{\Lambda}^{1}(G(\alpha), G(A))(G(\eta))$ is an exact sequence with all terms annihilated by the ideal a, it follows that $G\left(\eta^{\prime}\right)$ is exact and equal to $\operatorname{Ext}_{\Lambda}^{1}(G(\alpha), G(A))(G(\eta))$. Therefore the sequence is $F_{0}$-exact.

(b) Let $\eta: 0 \rightarrow A \rightarrow B \rightarrow C \rightarrow 0$ be an exact sequence such that $G(\eta): 0 \rightarrow$ $G(A) \rightarrow G(B) \rightarrow G(C) \rightarrow 0$ is $F_{0}$-exact. Let $\alpha: A \rightarrow A^{\prime}$ be a morphism in $\bmod \Lambda$. We have the following pushout diagram:

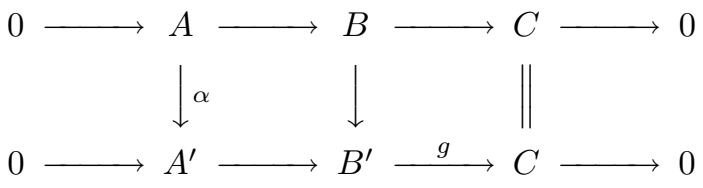

This diagram gives rise to the following exact commutative diagram:

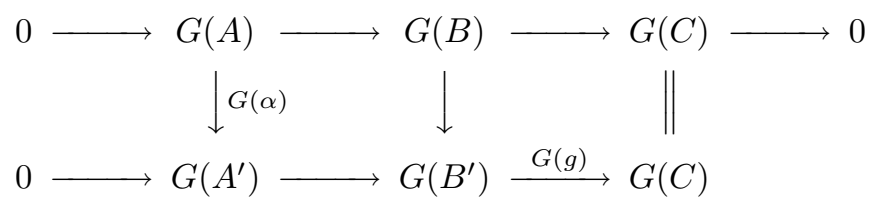

It follows directly from this commutative diagram that $G(g)$ is an epimorphism and $0 \rightarrow G\left(A^{\prime}\right) \rightarrow G\left(B^{\prime}\right) \rightarrow G(C) \rightarrow 0$ is exact. Since the $F_{0}$-exact sequences are closed under pushouts, this sequence is $F_{0}$-exact.

The statements for $G^{\prime}$ are proved similarly. 
It now follows from Lemma 1.9 that $F_{\left[G, F_{0}\right]}$ and $F_{\left[G^{\prime}, F_{0}\right]}$ are additive subbifunctors of $\operatorname{Ext}_{\Lambda}^{1}($,$) . Moreover, we have the following direct consequence of Proposition$ 1.10 and Corollary 1.5 .

Proposition 3.3. Let $\Lambda$ be an artin algebra with an ideal a, and let $G=$ $\operatorname{Hom}_{\Lambda}(\Lambda / \underline{\mathrm{a}}, \quad)$ and $G^{\prime}$ be as above, and let $F_{0}$ be a closed additive subbifunctor of $\operatorname{Ext}_{\Lambda}^{1}($,$) . Then the above functors F_{\left[G, F_{0}\right]}, F_{\left[G^{\prime}, F_{0}\right]}$ and $F_{\left[G, F_{0}\right]} \cap F_{\left[G^{\prime}, F_{0}\right]}$ define exact structures on $\bmod \Lambda$.

This can be applied to the special cases $F_{0}=(0)$ and $F_{0}=\operatorname{Ext}_{\Lambda}^{1}($,$) , that is,$ the $F_{0}$-exact sequences are the split ones and all exact sequences respectively.

In the case when $F_{0}=(0)$ we want to define a relation $\mathfrak{R}$ on the category $\bmod \Lambda$ with exact structure given by $F_{[G, 0]} \cap F_{\left[G^{\prime}, 0\right]}$, such that $\bmod \Lambda / \mathfrak{R}$ is exact with the induced structure.

Proposition 3.4. Let $\Lambda$ be an artin algebra with an ideal $\underline{\mathrm{a}}$, and let $G=$ $\operatorname{Hom}_{\Lambda}(\Lambda / \underline{\mathrm{a}}, \quad)$ and $G^{\prime}=\mathrm{id}_{\bmod \Lambda} / G$ be as before, with exact structure on $\bmod \Lambda$ given by $F=F_{[G, 0]} \cap F_{\left[G^{\prime}, 0\right]}$. Let $\mathfrak{R}$ be the relation on $\bmod \Lambda$ given by $\mathfrak{R}=\{f: A \rightarrow$ $\left.B \mid G(f)=0=G^{\prime}(f)\right\}$. Then $\mathcal{E}_{F} / \mathfrak{R}$ is an exact structure on $\bmod \Lambda / \mathfrak{R}$.

Proof. We want to show that $\mathfrak{R}$ satisfies conditions (I) and (II) of Proposition 1.11, with respect to the $F$-exact structure on $\bmod \Lambda$. Note that $f: A \rightarrow B$ is in $\mathfrak{R}$ if and only if there is a factorization $A \rightarrow G^{\prime}(A) \rightarrow G(B) \rightarrow B$.

We show that the four conditions (i)-(iv) of the proof of Proposition 1.11, which are equivalent to condition (I), are satisfied.

(i) Let $p: A \rightarrow B$ and $t: B \rightarrow C$ be such that $t p: A \rightarrow C$ is in $\Re$ and $p$ is a deflation. Then $G(p): G(A) \rightarrow G(B)$ and $G^{\prime}(p): G^{\prime}(A) \rightarrow G^{\prime}(B)$ are split epimorphisms. Since $G(t p)=G(t) G(p): G(A) \rightarrow G(C)$ is zero, it follows that $G(t)=0$. Since $G^{\prime}(t p)=G^{\prime}(t) G^{\prime}(p): G^{\prime}(A) \rightarrow G^{\prime}(C)$ is zero, it follows that $G^{\prime}(t)=0$. This shows that $\mathrm{t}$ is in $\mathfrak{R}$.

(ii) Let $s: A \rightarrow B$ and let $i: B \rightarrow C$ be an inflation, and assume that $i s: A \rightarrow C$ is in $\Re$. Then $G(i): G(B) \rightarrow G(C)$ and $G^{\prime}(i): G^{\prime}(B) \rightarrow G^{\prime}(C)$ are split monomorphisms. Since $G(i s)=G(i) G(s): G(A) \rightarrow G(C)$ is zero, it follows that $G(s)=0$. Since $G^{\prime}(i s)=G^{\prime}(i) G^{\prime}(s): G^{\prime}(A) \rightarrow G^{\prime}(C)$ is zero, it follows that $G^{\prime}(s)=0$. This shows that $s$ is in $\mathfrak{R}$.

(iii) Let $p: B \rightarrow C$ be a deflation and let $t: X \rightarrow C$ be in $\Re$. Consider

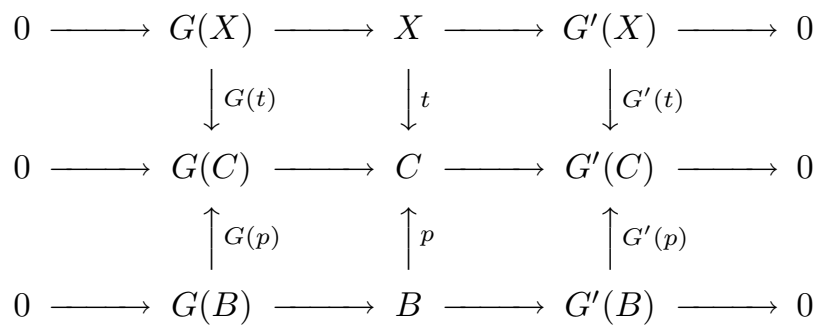

Then $G(p): G(B) \rightarrow G(C)$ is a split epimorphism, so choose $u: G(C) \rightarrow G(B)$ such that $G(p) u=\operatorname{id}_{G(C)}$. Since $t: X \rightarrow C$ is in $\mathfrak{R}$, we have a factorization of $t$ as $X \rightarrow G^{\prime}(X) \stackrel{t^{\prime}}{\rightarrow} G(C) \rightarrow C$, and consequently $s: X \rightarrow G^{\prime}(X) \stackrel{u t^{\prime}}{\rightarrow} G(B) \rightarrow B$. We have $s$ in $\mathfrak{R}$, and clearly $p s=t$. 
(iv) Let $i: A \rightarrow C$ be an inflation and $u: A \rightarrow Y$ in $\Re$. Consider

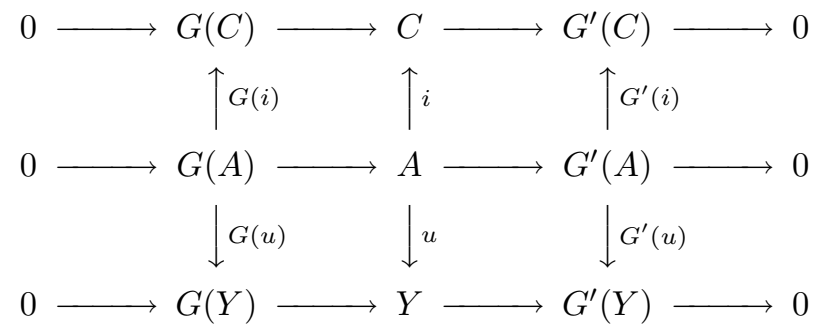

Then $G^{\prime}(i): G^{\prime}(A) \rightarrow G^{\prime}(C)$ is a split monomorphism, so choose $v: G^{\prime}(C) \rightarrow G^{\prime}(A)$ such that $s G^{\prime}(i)=\operatorname{id}_{G^{\prime}(A)}$. Since $u$ is in $\mathfrak{R}$, the morphism $u: A \rightarrow Y$ factors as $A \rightarrow G^{\prime}(A) \stackrel{u^{\prime}}{\rightarrow} G(Y) \rightarrow Y$. Define $v: C \rightarrow Y$ by $C \stackrel{u}{\rightarrow} G^{\prime}(C) \stackrel{u^{\prime} s}{\rightarrow} G(Y) \rightarrow Y$. Then $v$ is in $\mathfrak{R}$ and clearly $v i=u$.

(II) Let $X$ be any module in $\bmod \Lambda$ and $r$ a morphism in $\mathfrak{R}(X, X)$. Then $r$ is of the form $i_{X} r^{\prime} \pi_{X}$ for some morphism $r^{\prime}: G^{\prime}(X) \rightarrow G(X)$, where $i_{X}: G(X) \rightarrow X$ and $\pi_{X}: X \rightarrow G^{\prime}(X)$ are the natural inclusion and projection, respectively. It follows that $r^{2}=0$. Therefore $\left(\mathrm{id}_{X}+r\right)\left(\mathrm{id}_{X}-r\right)=\mathrm{id}_{X}$, and $\mathrm{id}_{X}+r$ is an isomorphism for all $X$ in $\bmod \Lambda$ and $r$ in $\mathfrak{R}(X, X)$. This finishes the proof.

We have the following immediate corollaries of Propositions 3.3 and 3.4.

Corollary 3.5. Let $\Lambda$ be an artin algebra with an ideal $\underline{\mathrm{a}}$, let $G$ and $G^{\prime}$ be as before and let $F_{0}$ be a closed additive subbifunctor of $\operatorname{Ext}_{\Lambda}^{1}($,$) . Then \bmod \Lambda$ with the exact structures induced from the subbifunctors $F_{\left[G, F_{0}\right]}, F_{\left[G^{\prime}, F_{0}\right]}$ and $F_{\left[G, F_{0}\right]} \cap F_{\left[G^{\prime}, F_{0}\right]}$ of $\operatorname{Ext}_{\Lambda}^{1}(,$,$) all have almost split pairs.$

Corollary 3.6. Let $\Lambda$ be an artin algebra with an ideal $\underline{\mathrm{a}}$, and let $G$ and $G^{\prime}$ be as before, with exact structure on $\bmod \Lambda$ given by $F=F_{[G, 0]} \cap F_{\left[G^{\prime}, 0\right]}$. Let $\mathfrak{R}$ be the relation on $\bmod \Lambda$ given by $\mathfrak{R}=\left\{f: A \rightarrow B \mid G(f)=0=G^{\prime}(f)\right\}$. Then $\bmod \Lambda / \mathfrak{R}$ with the exact structure induced from $F$ modulo the relation $\mathfrak{R}$ has almost split pairs.

In view of the above results it is evidently important to be able to calculate the relative projectives and relative injectives. Before we do this in some classes of examples we need to prove some elementary properties of the functor $\operatorname{Hom}_{\Lambda}(\Lambda / \underline{a}$,$) .$

3.2. Elementary properties of $\operatorname{Hom}_{\Lambda}(\Lambda / \underline{a}$,$) . Let G=\operatorname{Hom}_{\Lambda}(\Lambda / \underline{a}):, \bmod \Lambda \rightarrow$ $\bmod \Lambda$ and $G^{\prime}=\operatorname{id}_{\bmod \Lambda} / G$, as before. In this subsection we first observe that all additive left exact subfunctors of the identity are naturally isomorphic to $\operatorname{Hom}_{\Lambda}(\Lambda / \underline{a}$, for some ideal a, and give some elementary properties of these subfunctors of the identity.

Lemma 3.7. If $G$ is an additive left exact subfunctor of the identity functor, then

$$
G=\operatorname{ann}_{\underline{\mathrm{a}}}() \simeq \operatorname{Hom}_{\Lambda}(\Lambda / \underline{\mathrm{a}},)
$$

for an ideal $\underline{\mathrm{a}}$ in $\Lambda$.

Proof. We have that $D \circ G \circ D: \bmod \Lambda^{\mathrm{op}} \rightarrow \bmod \Lambda^{\mathrm{op}}$ is a right exact additive functor. Then $D \circ G \circ D \simeq-\otimes_{\Lambda} D G D(\Lambda)$. Since it is a quotient functor of the identity

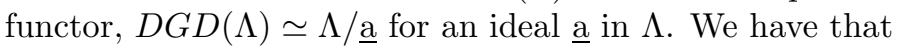

$$
G \simeq D\left(D() \otimes_{\Lambda} \Lambda / \underline{\mathrm{a}}\right) \simeq \operatorname{Hom}_{\Lambda}(\Lambda / \underline{\mathrm{a}},)
$$

by the adjoint isomorphism. This completes the proof of the lemma. 
Next we give some properties of additive left exact subfunctors of the identity functor that follow easily from the above characterization of these functors.

Proposition 3.8. Let a be an ideal in $\Lambda$, and let $G$ and $G^{\prime}$ be as before. Then we have the following.

(a) $G: \bmod \Lambda \rightarrow \bmod \Lambda$ a is a dense functor.

(b) $G^{2}=G$.

(c) $G^{\prime} \circ G=0$.

(d) $G \circ G^{\prime}=\operatorname{Hom}_{\Lambda}\left(\Lambda / \underline{\mathrm{a}}^{2},\right) / \operatorname{Hom}_{\Lambda}(\Lambda / \underline{\mathrm{a}}$,$) .$

(e) $\operatorname{Im} G^{\prime}$ is closed under submodules.

Proof. (a) Let $X$ be a $\Lambda$ /a-module. Then $G(X)=\operatorname{Hom}_{\Lambda}(\Lambda / \underline{\mathrm{a}}, X)=X$, and $G: \bmod \Lambda \rightarrow \bmod \Lambda / \underline{\mathrm{a}}$ is a dense functor.

(b) Since $G=\operatorname{ann}_{\underline{a}}($ ), this follows directly.

(c) Applying the exact sequence of functors

$$
0 \rightarrow G \rightarrow \operatorname{id}_{\bmod \Lambda} \rightarrow G^{\prime} \rightarrow 0
$$

to an object $G(X)$, we obtain the exact sequence

$$
0 \rightarrow G^{2}(X) \rightarrow G(X) \rightarrow G^{\prime} \circ G(X) \rightarrow 0 .
$$

By (b) we get that $G^{\prime} \circ G(X)=0$, hence $G^{\prime} \circ G=0$.

(d) Using the equality $G=\operatorname{Hom}_{\Lambda}(\Lambda / \underline{\mathrm{a}})=,\operatorname{ann}_{\underline{\mathbf{a}}}($ ), it is easy to see that for an arbitrary module $X$ in $\bmod \Lambda$

$$
G \circ G^{\prime}(X)=G(X / G(X))=\operatorname{Hom}_{\Lambda}\left(\Lambda / \underline{\mathrm{a}}^{2}, X\right) / G(X),
$$

since $\operatorname{ann}_{\underline{a}}\left(X / \operatorname{ann}_{\underline{a}}(X)\right)=\operatorname{ann}_{\underline{a}^{2}}(X) / \operatorname{ann}_{\underline{a}}(X)$. Hence, we get that

$$
G \circ G^{\prime}=\operatorname{Hom}_{\Lambda}\left(\Lambda / \underline{\mathrm{a}}^{2},\right) / G \text {. }
$$

(e) Let $M$ be in $\bmod \Lambda$ and $X$ a submodule of $G^{\prime}(M)$. Then we have the following commutative diagram:

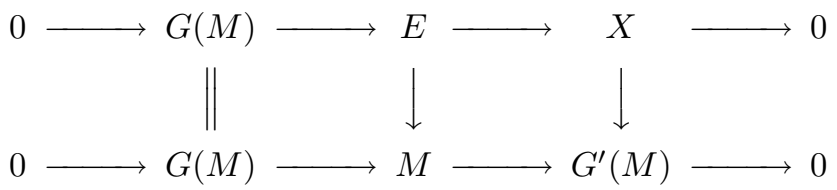

Applying $G$ to this diagram and using (b), it follows that $G(E)=G(M)$ and therefore $G^{\prime}(E)=X$.

These easy observations naturally lead to some interesting special cases, as we shall see later.

Corollary 3.9. Let $G=\operatorname{Hom}_{\Lambda}\left(\Lambda / \underline{a}\right.$, ) for an ideal a in $\Lambda$, and let $G^{\prime}=\operatorname{id}_{\bmod \Lambda} / G$.

(a) Then (i) $\underline{a}^{2}=(0)$, (ii) $G \circ G^{\prime}=G^{\prime}$ and (iii) $\left(G^{\prime}\right)^{2}=0$ are equivalent statements.

(b) Then (i) $\underline{\mathrm{a}}^{2}=\underline{\mathrm{a}}$, (ii) $G \circ G^{\prime}=0$ and (iii) $\left(G^{\prime}\right)^{2}=G^{\prime}$ are equivalent statements.

Proof. (a) Using the exact sequence of functors $0 \rightarrow G \rightarrow \operatorname{id}_{\bmod \Lambda} \rightarrow G^{\prime} \rightarrow 0$, the equivalence of (ii) and (iii) is immediate.

If $\underline{\mathrm{a}}^{2}=(0)$, we get from Proposition 3.8 (d) that $G \circ G^{\prime}=\operatorname{id}_{\bmod \Lambda} / G=G^{\prime}$. 
Conversely, assume that $\left(G^{\prime}\right)^{2}=0$. Then for a $\Lambda$-module $X$ we have that

$$
\begin{aligned}
(0)=G^{\prime} \circ G^{\prime}(X) & =G^{\prime}(X / G(X))=(X / G(X)) / G(X / G(X)) \\
& =(X / G(X)) /\left(\operatorname{Hom}_{\Lambda}\left(\Lambda / \underline{\mathrm{a}}^{2}, X\right) / G(X)\right) \simeq X / \operatorname{Hom}_{\Lambda}\left(\Lambda / \underline{\mathrm{a}}^{2}, X\right)
\end{aligned}
$$

It follows that $\operatorname{Hom}_{\Lambda}(\Lambda, X)=\operatorname{Hom}_{\Lambda}\left(\Lambda / \underline{\mathrm{a}}^{2}, X\right)=\operatorname{ann}_{\underline{\underline{a}}^{2}}(X)$ for all $\Lambda$-modules $X$ in $\bmod \Lambda$. In particular this is true for $X=\Lambda$, which implies that $\Lambda=\operatorname{ann}_{\underline{a}^{2}}(\Lambda)$ and therefore $\underline{a}^{2}=(0)$.

(b) Using the exact sequence of functors $0 \rightarrow G \rightarrow \operatorname{id}_{\bmod \Lambda} \rightarrow G^{\prime} \rightarrow 0$, the equivalence of (ii) and (iii) is immediate.

If $\underline{\mathrm{a}}^{2}=\underline{\mathrm{a}}$, then it follows from Proposition 3.8 (d) that $G \circ G^{\prime}=0$.

Conversely, assume that $G \circ G^{\prime}=0$. Hence $\operatorname{Hom}_{\Lambda}\left(\Lambda / \underline{\mathrm{a}}^{2}, X\right)=\operatorname{Hom}_{\Lambda}(\Lambda / \underline{\mathrm{a}}, X)$, or equivalently $\operatorname{ann}_{\underline{a}^{2}}(X)=\operatorname{ann}_{\underline{a}}(X)$, for all $\Lambda$-modules $X$ in mod $\Lambda$. In particular this is true for $X=\Lambda / \underline{a}^{2}$, from which it follows easily that $\underline{\mathrm{a}} \subset \underline{\mathrm{a}}^{2}$ and therefore $\underline{\mathrm{a}}=\underline{\mathrm{a}}^{2}$.

3.3. The projectives and injectives of the subbifunctors $F_{[G, 0]}$ and $F_{\left[G^{\prime}, 0\right]}$. Let $G=\operatorname{Hom}_{\Lambda}(\Lambda / \underline{\mathrm{a}}):, \bmod \Lambda \rightarrow \bmod \Lambda$ for an ideal $\underline{\mathrm{a}}$ in $\Lambda$ and $G^{\prime}=\operatorname{id}_{\bmod \Lambda} / G$, where $\operatorname{id}_{\bmod \Lambda}: \bmod \Lambda \rightarrow \bmod \Lambda$ is the identity functor. Denote by $F_{[G]}$ the subbifunctor $F_{[G, 0]}$ and by $F_{\left[G^{\prime}\right]}$ the subbifunctor $F_{\left[G^{\prime}, 0\right]}$ of $\operatorname{Ext}_{\Lambda}^{1}($,$) . By Proposition 3.4$ they are both additive subbifunctors. Now we describe the projectives and injectives for these subbifunctors of $\operatorname{Ext}_{\Lambda}^{1}($,$) , in general for the former and in some$ special cases for the latter.

Since $G=G^{2}$, the functor $G$ has the following property. For any homomorphism $f: G(X) \rightarrow Y$ for $X$ and $Y$ in $\bmod \Lambda$ the image $\operatorname{Im} f$ is contained in $G(Y)$. This is the crucial observation needed in the characterization of the subbifunctor $F_{[G]}$ of $\operatorname{Ext}_{\Lambda}^{1}($,$) below.$

Proposition 3.10. Let $G=\operatorname{Hom}_{\Lambda}(\Lambda / \underline{\mathrm{a}}$,$) for an ideal a in \Lambda$ and let $F_{[G]}$ be as above. Then

$$
F_{[G]}=F_{\operatorname{Im} G}=F_{\bmod \Lambda / \underline{a}} .
$$

In particular,

$$
\mathcal{P}\left(F_{[G]}\right)=\mathcal{P}(\Lambda) \cup \operatorname{Im} G=\mathcal{P}(\Lambda) \cup \bmod \Lambda / \underline{\text { a }} .
$$

Proof. Since $\operatorname{Im} G=\bmod \Lambda / \underline{\mathrm{a}}$, we have $F_{\operatorname{Im} G}=F_{\bmod \Lambda / \underline{a}}$.

Assume that $\eta: 0 \rightarrow A \rightarrow B \stackrel{g}{\rightarrow} C \rightarrow 0$ is $F_{[G]}$-exact. Let $X$ be a module in $\operatorname{add}(\operatorname{Im} G)=\operatorname{Im} G$ and $f: X \rightarrow C$ a homomorphism. Since $G^{2}=G$, we have that $X=G(X)$ and therefore $\operatorname{Im} f$ is contained in $G(C)$. Since $G(\eta)$ is split exact, the homomorphism $f$ factors through $g$. This shows that $F_{[G]}$ is contained in $F_{\operatorname{Im} G}$.

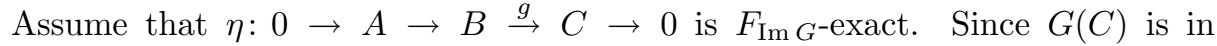
$\operatorname{Im} G$, there exists a homomorphism $h: G(C) \rightarrow B$ such that $g \circ h=i_{C}$, where $i_{C}: G(C) \rightarrow C$ is the natural inclusion. The image $\operatorname{Im} h$ is contained in $G(B)$. Hence $g \circ i_{B} \circ h=i_{C} \circ G(g) \circ h=i_{C} \circ \operatorname{id}_{G(C)}$. Since $i_{C}$ is a monomorphism, $G(g) \circ h=\operatorname{id}_{G(C)}$ and hence $G(\eta)$ is split exact. It follows that $F_{\operatorname{Im} G}=F_{\bmod \Lambda / \underline{a}}$ is contained in $F_{[G]}$, and therefore $F_{[G]}=F_{\operatorname{Im} G}$. Moreover, $\mathcal{P}\left(F_{[G]}\right)=\mathcal{P}(\Lambda) \cup \operatorname{Im} G=$ $\mathcal{P}(\Lambda) \cup \bmod \Lambda / \underline{a}$.

It seems not so easy to get a description of the relative projectives or equivalently the relative injectives for the subbifunctor $F_{\left[G^{\prime}\right]}$ of $\operatorname{Ext}_{\Lambda}^{1}($,$) in general. Below we$ give a description in a special case. 
Proposition 3.11. Let $G=\operatorname{Hom}_{\Lambda}(\Lambda / \underline{\mathrm{a}}$,$) for an ideal \underline{\mathrm{a}}$ in $\Lambda$ with $\underline{\mathrm{a}}^{2}=\underline{\mathrm{a}}$. Then

$$
F_{\left[G^{\prime}\right]}=F^{\operatorname{add}\left(\operatorname{Im} G^{\prime}\right)} \cap F_{\Lambda / \underline{a}}=F^{\operatorname{add}\left(\operatorname{Im} G^{\prime}\right) \cup \operatorname{add} D \operatorname{Tr}_{\Lambda}(\Lambda / \underline{a})} .
$$

In particular, we have that

$$
\mathcal{I}\left(F_{\left[G^{\prime}\right]}\right)=\mathcal{I}(\Lambda) \cup \operatorname{add}\left(\operatorname{Im} G^{\prime}\right) \cup \operatorname{add} D \operatorname{Tr}_{\Lambda}(\Lambda / \underline{a})
$$

and

$$
\mathcal{P}\left(F_{\left[G^{\prime}\right]}\right)=\mathcal{P}(\Lambda) \cup \operatorname{add} \operatorname{Tr}_{\Lambda} D\left(\operatorname{Im} G^{\prime}\right) \cup \operatorname{add} \Lambda / \underline{\text { a. }}
$$

Proof. Assume that $\eta: 0 \rightarrow A \stackrel{f}{\rightarrow} B \rightarrow C \rightarrow 0$ is $F_{\left[G^{\prime}\right]}$-exact. In particular, the complex $0 \rightarrow G(A) \rightarrow G(B) \rightarrow G(C) \rightarrow 0$ is exact, hence the sequence $\eta$ is $F_{\Lambda / \underline{a}^{-}}$ exact.

Now we show that $\eta$ is $F^{\text {add }\left(\operatorname{Im} G^{\prime}\right)}$-exact. Let $X$ be a module in $\operatorname{add}\left(\operatorname{Im} G^{\prime}\right)$ and $g: A \rightarrow X$ a homomorphism. Then there exists $X^{\prime}$ in $\bmod \Lambda$ such that $X$ is a direct summand of $G^{\prime}\left(X^{\prime}\right)$. Let $g^{\prime}: A \rightarrow G^{\prime}\left(X^{\prime}\right)$ be the homomorphism induced by $g$. Then we have the following commutative diagram:

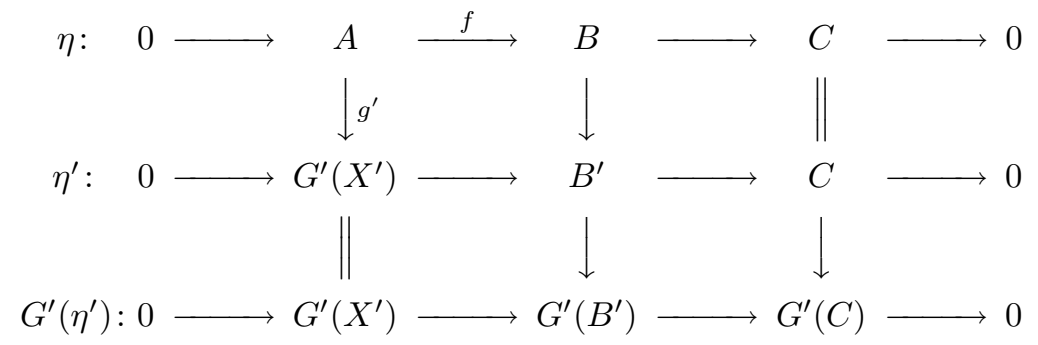

The sequence $\eta^{\prime}: 0 \rightarrow G^{\prime}\left(X^{\prime}\right) \rightarrow B^{\prime} \rightarrow C \rightarrow 0$ is the pushout of $\eta$ using the homomorphism $g^{\prime}$; hence $\eta^{\prime}$ is $F_{\left[G^{\prime}\right]}$-exact. Therefore $G^{\prime}\left(\eta^{\prime}\right)$ is split exact, which is the last row in the diagram above. It follows that the homomorphism $g^{\prime}$ factors through $f$ and therefore also $g$ factors through $f$. This shows that $F_{\left[G^{\prime}\right]}$ is contained

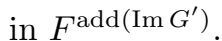

It follows from the above that $F_{\left[G^{\prime}\right]}$ is contained in $F^{\text {add }\left(\operatorname{Im} G^{\prime}\right)} \cap F_{\Lambda / \underline{a}}$, which is

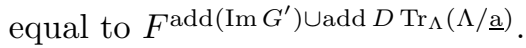

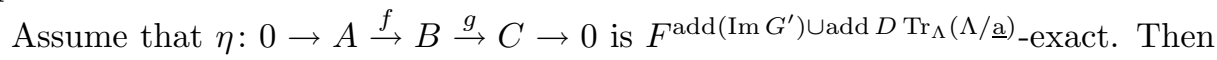
the sequence $G(\eta)$ is exact, and therefore $G^{\prime}(\eta)$ is exact by Lemma 3.1.

Then we have the following exact commutative diagram:

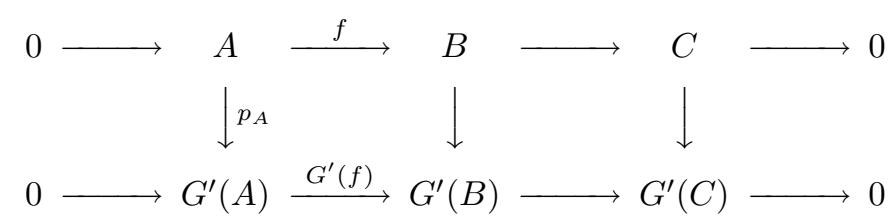

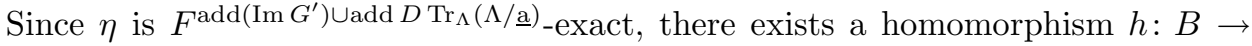
$G^{\prime}(A)$ such that $h \circ f=p_{A}$. Applying the functor $G^{\prime}$ we get that $G^{\prime}(h) \circ G^{\prime}(f)=$ $\operatorname{id}_{G^{\prime}(A)}$; hence $G^{\prime}(\eta)$ is split exact and $\eta$ is $F_{\left[G^{\prime}\right]}$-exact. This shows that the subbifunctor $F^{\operatorname{add}\left(\operatorname{Im} G^{\prime}\right) \cup \operatorname{Uadd} D \operatorname{Tr}_{\Lambda}(\Lambda / \underline{a})}$ is contained in $F_{\left[G^{\prime}\right]}$.

We have now shown that $F_{\left[G^{\prime}\right]}=F^{\operatorname{add}\left(\operatorname{Im} G^{\prime}\right) \cup \text { add } D \operatorname{Tr}_{\Lambda}(\Lambda / \text { a })}$. It follows from this that $\mathcal{I}\left(F_{\left[G^{\prime}\right]}\right)=\mathcal{I}(\Lambda) \cup \operatorname{add}\left(\operatorname{Im} G^{\prime}\right) \cup \operatorname{add} D \operatorname{Tr}_{\Lambda}(\Lambda / \underline{\text { a }})$. 
Next we describe a subcategory which always is contained in the relative injectives for $F_{\left[G^{\prime}\right]}$.

Proposition 3.12. Let $\mathcal{X}=\left\{X \in \bmod \Lambda \mid G^{\prime}(X)=X\right\}$. Then $\mathcal{X} \subset \mathcal{I}\left(F_{\left[G^{\prime}\right]}\right)$ and $F_{\left[G^{\prime}\right]} \subset F^{\mathcal{X}}$.

Proof. Let $X$ be a module in $\mathcal{X}$ and $\eta: 0 \rightarrow X \stackrel{f}{\rightarrow} Y \rightarrow Z \rightarrow 0$ an $F_{\left[G^{\prime}\right]}$-exact sequence. Then we have the following exact commutative diagram:

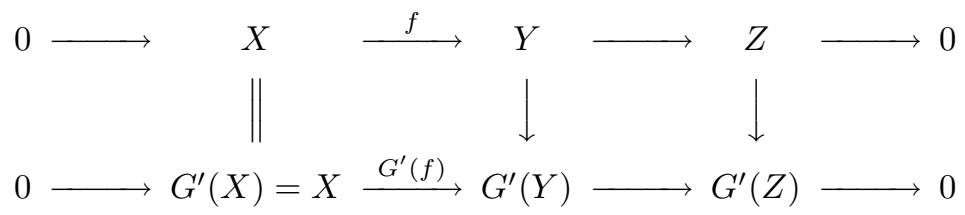

Since $G^{\prime}(\eta)$ is split exact, $f$ is a split monomorphism and $\eta$ is also split exact. Therefore $\mathcal{X} \subset \mathcal{I}\left(F_{\left[G^{\prime}\right]}\right)$ and $F_{\left[G^{\prime}\right]} \subset F^{\mathcal{X}}$.

We are mainly interested in calculating the subbifunctor $F=F_{[G]} \cap F_{\left[G^{\prime}\right]}$ of $\operatorname{Ext}_{\Lambda}^{1}($,$) . Then we have that \mathcal{P}\left(F_{[G]}\right) \cup \mathcal{P}\left(F_{\left[G^{\prime}\right]}\right)$ is contained in $\mathcal{P}(F)$. Hence, $\operatorname{Im} G$ is contained in $\mathcal{P}(F)$ by Proposition 3.10. If $\mathcal{X}$ and $\mathcal{Y}$ are two subcategories of $\bmod \Lambda$ and $F_{\mathcal{X}}$ and $F_{\mathcal{Y}}$ are the two corresponding subbifunctors of $\operatorname{Ext}_{\Lambda}^{1}($,$) ,$

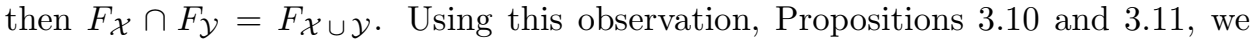
obtain the following corollary.

Corollary 3.13. Suppose $G=\operatorname{Hom}_{\Lambda}(\Lambda / \underline{\mathrm{a}}$,$) for an ideal \underline{\mathrm{a}}$ in $\Lambda$ with $\underline{\mathrm{a}}^{2}=\underline{\mathrm{a}}$. Then

$$
F_{[G]} \cap F_{\left[G^{\prime}\right]}=F_{\operatorname{Im} G} \cap F^{\operatorname{add}\left(\operatorname{Im} G^{\prime}\right) \cup \operatorname{add} D \operatorname{Tr}_{\Lambda}(\Lambda / \underline{\mathrm{a}})}=F^{\operatorname{add}\left(\operatorname{Im} G^{\prime}\right) \cup D \operatorname{Tr}_{\Lambda}(\bmod \Lambda / \underline{\mathrm{a}})} .
$$

In particular,

$$
\mathcal{I}\left(F_{[G]} \cap F_{\left[G^{\prime}\right]}\right)=\mathcal{I}(\Lambda) \cup \operatorname{add}\left(\operatorname{Im} G^{\prime}\right) \cup D \operatorname{Tr}_{\Lambda}(\bmod \Lambda / \underline{\mathrm{a}})
$$

and

$$
\mathcal{P}\left(F_{[G]} \cap F_{\left[G^{\prime}\right]}\right)=\mathcal{P}(\Lambda) \cup \operatorname{Tr}_{\Lambda} D \operatorname{Im} G^{\prime} \cup \bmod \Lambda / \underline{\text { a. }} .
$$

Suppose that $G=\operatorname{Hom}_{\Lambda}(\Lambda / \underline{\mathrm{a}}, \quad)$ for some ideal $\underline{\mathrm{a}}$ in $\Lambda$. Let $\underline{\mathrm{b}}$ be the ideal given by $G(\Lambda)=\{\lambda \in \Lambda \mid \underline{\mathrm{a}} \lambda=(0)\}$. For any $\Lambda$-module $M$ in $\bmod \Lambda$ we have that $b m$ is in $G(M)$ for any $b$ in $\underline{\mathrm{b}}$ and $m$ in $M$, since for any $a$ in a we have that $a \cdot(b m)=(a b) m=0$. Therefore $\underline{\mathrm{b}} M \subset G(M)$, and hence we get an induced functor

$$
G^{\prime}: \bmod \Lambda \rightarrow \bmod \Lambda / \underline{\mathrm{b}} \text {. }
$$

A $\Lambda / \underline{b}$-module $M$ is semisimple if and only if $M$ is a semisimple $\Lambda$-module. Hence, $\Lambda / \underline{b}$ is a semisimple ring if and only if $\underline{r}$ is contained in $\underline{b}$ or equivalently $\underline{a}$ is semisimple as a right $\Lambda$-module. Using these observations, we have the following description of the intersection $F_{[G]} \cap F_{\left[G^{\prime}\right]}$ in this case.

Proposition 3.14. Suppose that $G=\operatorname{Hom}_{\Lambda}(\Lambda / \underline{\mathrm{a}}$, ) for some twosided ideal a in

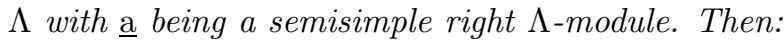
(a) $F_{\left[G^{\prime}\right]}=F_{\Lambda / \underline{a}}$.
(b) $F_{[G]} \cap F_{\left[G^{\prime}\right]}=F_{[G]}=F_{\bmod \Lambda / \underline{a}}$.

Proof. (a) From the above we have that $G^{\prime}: \bmod \Lambda \rightarrow \bmod \Lambda / \underline{b}$, where $\underline{b}=\{\lambda \in$ $\Lambda \mid \underline{\mathrm{a}} \lambda=(0)\}$ and $\Lambda / \underline{\mathrm{b}}$ is semisimple. Therefore, given an exact sequence $\eta: 0 \rightarrow$ $A \rightarrow B \rightarrow C \rightarrow 0$, then $G^{\prime}(\eta)$ is split exact if and only if $G(\eta)$ is exact. Hence, $F_{\left[G^{\prime}\right]}=F_{\Lambda / \underline{a}}$. 
(b) This follows immediately from (a) and Proposition 3.10, since under our assumptions $F_{[G]} \subset F_{\left[G^{\prime}\right]}$.

The next result characterizes when a module $X$ is in $\mathcal{I}\left(F_{[G]} \cap F_{\left[G^{\prime}\right]}\right)$.

Proposition 3.15. A module $X$ is in $\mathcal{I}\left(F_{[G]} \cap F_{\left[G^{\prime}\right]}\right)$ if and only if for every $F_{[G]} \cap F_{\left[G^{\prime}\right]}$-exact sequence $0 \rightarrow A \rightarrow B \stackrel{g}{\rightarrow} C \rightarrow 0$ and homomorphisms $h: G(C) \rightarrow$ $X$ and $h^{\prime}: B \rightarrow X$ such that $h \circ G(g)=h^{\prime} \circ i_{B}$ there exists a homomorphism $t: C \rightarrow X$ satisfying $h=t_{\circ} i_{C}$, where $i_{M}: G(M) \rightarrow M$ is the natural inclusion.

Proof. Let $F=F_{[G]} \cap F_{\left[G^{\prime}\right]}$. Let $\eta: 0 \rightarrow A \stackrel{f}{\rightarrow} B \stackrel{g}{\rightarrow} C \rightarrow 0$ be an $F$-exact sequence. Then we have the following commutative diagram.

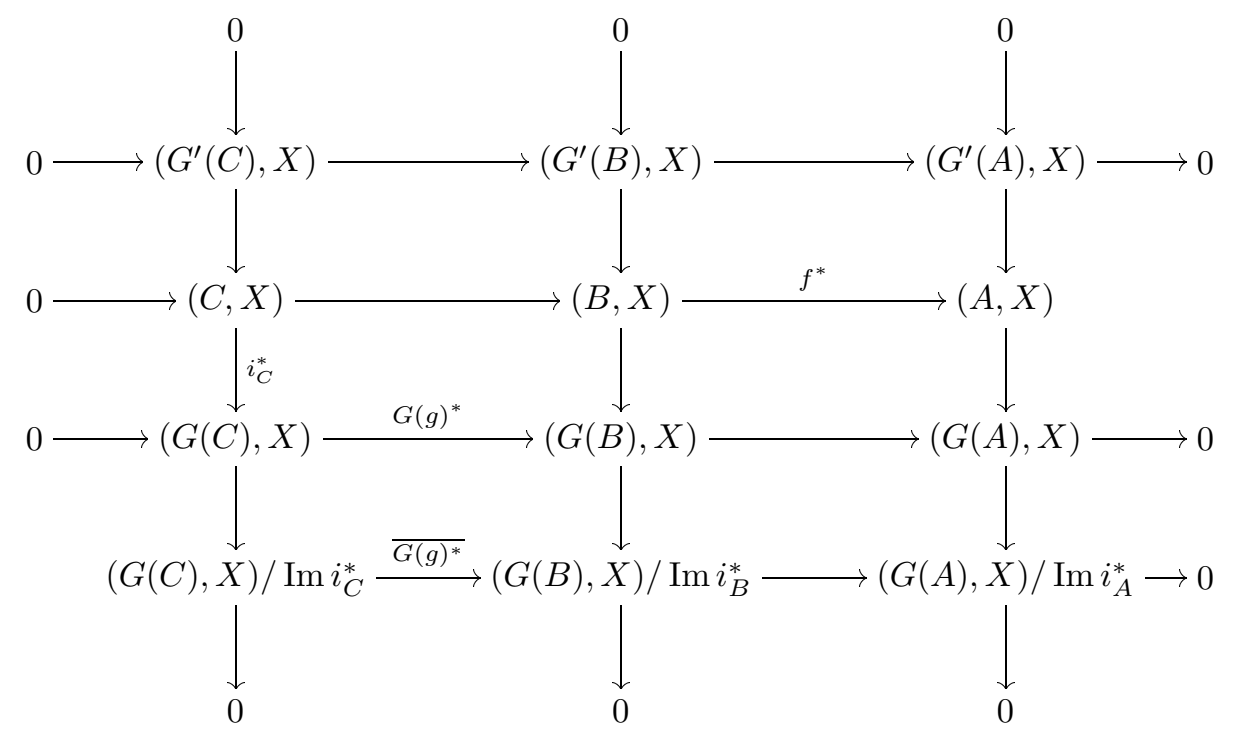

It is easy to see that $f^{*}$ is an epimorphism if and only if $\overline{G(g)^{*}}$ is a monomorphism. Hence $X$ is relative injective if and only if $\overline{G(g)^{*}}$ is a monomorphism for all $F$-exact sequences $\eta$. The morphism $\overline{G(g)^{*}}$ is a monomorphism if and only if, whenever we are given $h: G(C) \rightarrow X$ such that $h \circ G(g)=h^{\prime} \circ i_{B}$ for a homomorphism $h^{\prime}: B \rightarrow X$, then there exists a homomorphism $t: C \rightarrow X$ such that $h=t_{\circ} i_{C}$. This completes the proof of the proposition.

An easy corollary that we use next is the following.

Corollary 3.16. The set

$\left\{X \in \bmod \Lambda \mid 0 \rightarrow\left(G^{\prime}(M), X\right) \rightarrow(M, X) \rightarrow(G(M), X) \rightarrow 0\right.$ is exact for all $\left.M\right\}$ is contained in $\mathcal{I}\left(F_{[G]} \cap F_{\left[G^{\prime}\right]}\right)$.

We end with an observation for the situation $\underline{\mathrm{a}}^{2}=\underline{\mathrm{a}}$, which says that the set of sequences $\eta_{M}: 0 \rightarrow G(M) \rightarrow M \rightarrow G^{\prime}(M) \rightarrow 0$ is in some sense a generating set for $F=F_{[G]} \cap F_{\left[G^{\prime}\right]}$.

Lemma 3.17. Suppose $\underline{\mathrm{a}}^{2}=\underline{\mathrm{a}}$ and let $F=F_{[G]} \cap F_{\left[G^{\prime}\right]}$. Then we have the following.

(a) The sequences $\eta_{M}: 0 \rightarrow G(M) \rightarrow M \rightarrow G^{\prime}(M) \rightarrow 0$ for $M$ in $\bmod \Lambda$ are F-exact. 
(b) $\left\{X \in \bmod \Lambda \mid 0 \rightarrow\left(G^{\prime}(M), X\right) \rightarrow(M, X) \rightarrow(G(M), X) \rightarrow 0\right.$ is exact for all $M\}=\mathcal{I}(F)$.

Proof. (a) This follows directly from the fact that $G G^{\prime}=0$ and $G^{\prime} G=0$.

(b) This follows directly from (a) and Corollary 3.16.

3.4. Enough projectives and injectives. Throughout the subsection, let $G=$ $\operatorname{Hom}_{\Lambda}(\Lambda / \underline{\mathrm{a}}$,$) for an ideal \underline{\mathrm{a}}$ in $\Lambda, G^{\prime}=\operatorname{id}_{\bmod \Lambda} / G$ and $\underline{\mathrm{b}}=G(\Lambda)$. Now we investigate when the subbifunctors $F_{[G]}, F_{\left[G^{\prime}\right]}$ and their intersection have enough projectives and/or injectives. We recall that $F$ has enough projectives if for each $C$ in $\bmod \Lambda$ there is an $F$-exact sequence $0 \rightarrow K \rightarrow P \rightarrow C \rightarrow 0$, where $P$ is $F$-projective.

We start our investigation by considering the subbifunctor $F_{[G]}$, where we have a complete answer.

Proposition 3.18. The subcategory $\operatorname{Im} G$ is functorially finite in $\bmod \Lambda$. In particular, $F_{[G]}=F_{\bmod \Lambda / \underline{a}}$ has enough projectives and injectives.

Proof. This follows directly from the definitions (see [ASo, Corollary 1.13]).

The situation for the subbifunctor $F_{\left[G^{\prime}\right]}$ is more complicated. Here we only have partial results for the situation when $\underline{\mathrm{a}}^{2}=\underline{\mathrm{a}}$. First we point out what is true in general for this case.

Proposition 3.19. Suppose that $\underline{\mathrm{a}}^{2}=\underline{\mathrm{a}}$. Then we have the following.

(a) The subcategory $\operatorname{Im} G^{\prime}$ of $\bmod \Lambda / \underline{b}$ is closed under submodules and extensions. In particular, $\operatorname{Im} G^{\prime}$ is a resolving subcategory of $\bmod \Lambda / \underline{\mathrm{b}}$.

(b) The functor $G^{\prime}: \bmod \Lambda \rightarrow \operatorname{Im} G^{\prime}$ is a left adjoint of the inclusion functor $I: \operatorname{Im} G^{\prime} \rightarrow \bmod \Lambda$, and $\operatorname{Im} G^{\prime}$ is covariantly finite in $\bmod \Lambda$. In particular, $F_{\left[G^{\prime}\right]}$ has enough injectives.

Proof. Assume that $\underline{\mathrm{a}}^{2}=\underline{\mathrm{a}}$. Then we have already seen that $\left(G^{\prime}\right)^{2}=G^{\prime}$, or equivalently that $G G^{\prime}=0$. Hence, a module $X$ is in $\operatorname{Im} G^{\prime}$ if and only if $G(X)=(0)$.

(a) By Proposition $3.8 \operatorname{Im} G^{\prime}$ is closed under submodules.

If $0 \rightarrow A \rightarrow B \rightarrow C \rightarrow 0$ is exact with $A$ and $C$ in $\operatorname{Im} G^{\prime}$, then, because $G$ is left exact, it follows directly that $G(B)=(0)$, and therefore $B$ is in $\operatorname{Im} G^{\prime}$.

Since $G^{\prime}(\Lambda)=\Lambda / \underline{\mathrm{b}}$, it follows immediately from what we have shown that $\operatorname{Im} G^{\prime}$ is a resolving subcategory of $\bmod \Lambda / \underline{b}$.

(b) Let $I$ be the inclusion functor $I: \operatorname{Im} G^{\prime} \rightarrow \bmod \Lambda$. Let $X$ be in $\bmod \Lambda$, $Y$ in $\operatorname{Im} G^{\prime}$ and $f: X \rightarrow Y$ a homomorphism. Consider the natural projection $\pi_{X}: X \rightarrow G^{\prime}(X)$. Since $\left.\operatorname{Im} f\right|_{G(X)}$ is contained in $G(Y)=(0)$, we have that $G(X)$ is contained in Ker $f$. This implies that $f$ factors through $\pi_{X}$; that is, $f=\bar{f} \pi_{X}$ for a morphism $\bar{f}: G^{\prime}(X) \rightarrow Y$. Define

$$
\varphi: \operatorname{Hom}_{I^{\prime} G^{\prime}}\left(G^{\prime}(X), Y\right) \rightarrow \operatorname{Hom}_{\Lambda}(X, I(Y))
$$

by $\varphi(g)=g \pi_{X}$ and

$$
\psi: \operatorname{Hom}_{\Lambda}(X, I(Y)) \rightarrow \operatorname{Hom}_{\operatorname{Im} G^{\prime}}\left(G^{\prime}(X), Y\right)
$$

by $\psi(f)=\bar{f}$. It is straightforward to see that these morphisms are inverse natural isomorphisms. Hence $\left(G^{\prime}, I\right)$ is an adjoint pair, and $\operatorname{Im} G^{\prime}$ is therefore covariantly finite in $\bmod \Lambda$ by $[\mathrm{AR}]$.

Since $F_{\left[G^{\prime}\right]}=F^{\mathcal{I}(\Lambda) \cup \operatorname{Im} G^{\prime}}$, it follows immediately from [ASo, Theorem 1.12] that $F_{\left[G^{\prime}\right]}$ has enough injectives. 
Using the fact that the union of two covariantly finite subcategories of mod $\Lambda$ is again covariantly finite, we have the following immediate corollary of the two previous results when $\underline{\mathrm{a}}^{2}=\underline{\mathrm{a}}$.

Corollary 3.20. Suppose $\underline{\mathrm{a}}^{2}=\underline{\mathrm{a}}$. Then the subbifunctor $F=F_{[G]} \cap F_{\left[G^{\prime}\right]}$ has enough injectives, and $\mathcal{I}(F)=\mathcal{I}(\Lambda) \cup D \operatorname{Tr}_{\Lambda}(\bmod \Lambda / \underline{\mathrm{a}}) \cup \operatorname{add} \operatorname{Im} G^{\prime}$.

Consider the exact sequence $0 \rightarrow \underline{\mathrm{a}} \rightarrow \Lambda \rightarrow \Lambda / \underline{\mathrm{a}} \rightarrow 0$ and apply for $M$ in $\bmod \Lambda$ the functor $\operatorname{Hom}_{\Lambda}(, M)$. This gives the exact sequence

$$
0 \rightarrow G(M) \rightarrow M \stackrel{\alpha}{\rightarrow}(\underline{\mathrm{a}}, M) \rightarrow \operatorname{Ext}_{\Lambda}^{1}(\Lambda / \underline{\mathrm{a}}, M) \rightarrow 0,
$$

where $\operatorname{Im} \alpha=G^{\prime}(M)$. Using this sequence, we have the following.

Proposition 3.21. Suppose $\underline{\mathrm{a}}^{2}=\underline{\mathrm{a}}$. Then the following are equivalent.

(a) $G^{\prime}$ is a right exact functor.

(b) $\Lambda / \underline{\text { a }}$ is a projective $\Lambda$-module.

(c) $G^{\prime}$ is an exact functor.

(d) $\operatorname{Im} G^{\prime}=\bmod \Lambda / \underline{b}$.

Furthermore, if any of these equivalent conditions is satisfied, then there exists an idempotent $e$ in $\Lambda$ such that

$$
\begin{gathered}
\Lambda \simeq\left(\begin{array}{cc}
e \Lambda e & 0 \\
(1-e) \Lambda e & (1-e) \Lambda(1-e))
\end{array}\right), \\
\underline{\mathrm{a}}=\left(\begin{array}{cc}
e \Lambda e & 0 \\
(1-e) \Lambda e & 0
\end{array}\right) \quad \text { and } \underline{\mathrm{b}}=\left(\begin{array}{cc}
0 & 0 \\
(1-e) \Lambda e & (1-e) \Lambda(1-e)
\end{array}\right) .
\end{gathered}
$$

Proof. (a) implies (b). Suppose $G^{\prime}$ is a right exact functor. Let $0 \rightarrow A \rightarrow B \rightarrow$ $C \rightarrow 0$ be an exact sequence in $\bmod \Lambda$. Then we have the following commutative diagram:

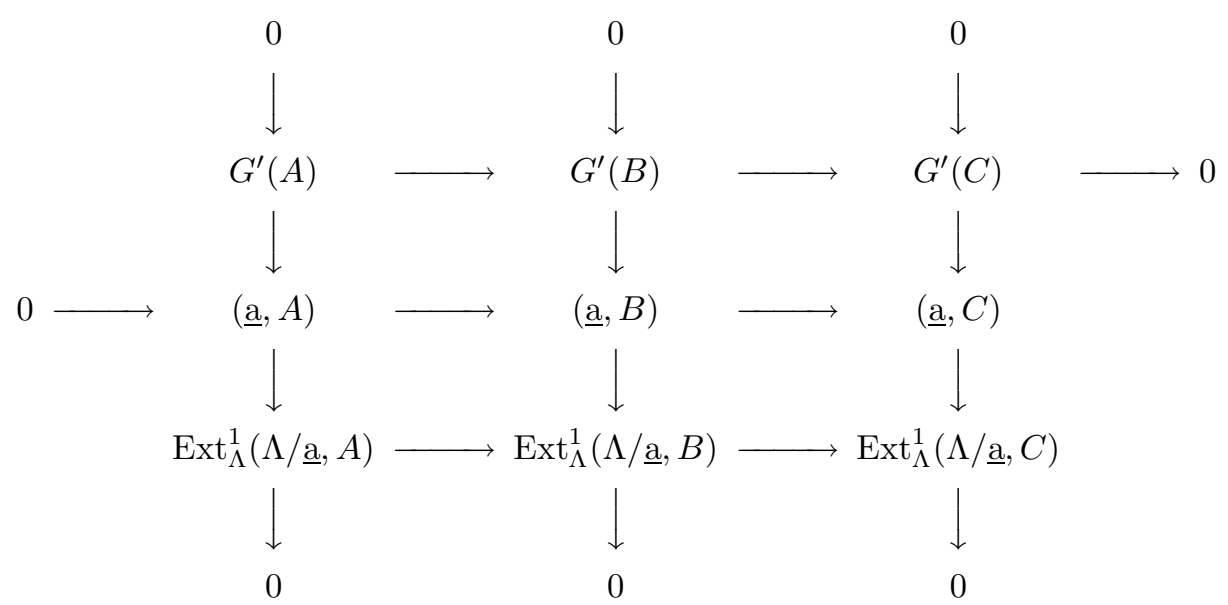

By assumption the two upper rows are exact, and therefore by the Snake Lemma the connecting homomorphism $\delta:(\Lambda / \underline{\mathrm{a}}, C) \rightarrow \operatorname{Ext}_{\Lambda}^{1}(\Lambda / \underline{\mathrm{a}}, A)$ is zero. Hence, $\Lambda / \underline{\mathrm{a}}$ is a projective $\Lambda$-module.

(b) implies (c). Suppose $\Lambda / \underline{a}$ is a projective $\Lambda$-module. Then $\underline{a}$ is also a projective $\Lambda$-module and it follows that $G^{\prime}=\operatorname{Hom}_{\Lambda}(\underline{\text { a }}$,$) , which is an exact functor.$

(c) implies (d). Suppose $G^{\prime}$ is an exact functor. Then $\Lambda / \underline{\mathrm{a}}$ is a projective $\Lambda$ module and therefore $\underline{a}$ is a direct summand of $\Lambda$. Hence there exists an idempotent 
$e$ such that $\underline{\mathrm{a}}=\Lambda e$. Since $\underline{\mathrm{a}}$ is a twosided ideal, $\underline{\mathrm{a}}=\Lambda e \Lambda$. We claim that $\underline{\mathrm{b}}=G(\Lambda)=$ $\Lambda(1-e) \Lambda$. Clearly we have that $(1-e) \Lambda \subset \underline{\mathrm{b}}$, since $\underline{\mathrm{a}}(1-e) \Lambda=\Lambda e(1-e) \Lambda=(0)$. Let $x$ be in $\underline{\mathrm{b}}$. Then $x=e x+(1-e) x$. Since $e$ is in $\underline{\mathrm{a}}$, it follows that $x=(1-e) x$ and therefore $\underline{\mathrm{b}}=(1-e) \Lambda=\Lambda(1-e) \Lambda$. Moreover, we have that $\underline{\mathrm{a}}+\underline{\mathrm{b}}=\Lambda$.

Let $X$ be an arbitrary $\Lambda / \underline{b}$-module viewed as a $\Lambda$-module. Since $G(X)=\{x \in$ $X \mid \underline{\mathrm{a}} x=(0)\}$ and $X$ is a $\Lambda / \underline{\mathrm{b}}$-module, we have that $(0)=(\underline{\mathrm{a}}+\underline{\mathrm{b}}) G(X)=\Lambda G(X)=$ $G(X)$. This shows that $\bmod \Lambda / \underline{\mathrm{b}}$ is contained in $\operatorname{Im} G^{\prime}$; hence $\operatorname{Im} G^{\prime}=\bmod \Lambda / \underline{\mathrm{b}}$.

(d) implies (a). Suppose $\operatorname{Im} G^{\prime}=\bmod \Lambda / \underline{b}$. From the previous result we have that $G^{\prime}: \bmod \Lambda \rightarrow \operatorname{Im} G^{\prime}$ is a left adjoint of the inclusion functor $I: \bmod \Lambda / \underline{\mathrm{b}}=$ $\operatorname{Im} G^{\prime} \rightarrow \bmod \Lambda$. Then $G^{\prime}$ is a right exact functor.

Suppose now that one of the equivalent conditions in (a)-(d) is true. Then we have found an idempotent $e$ in the proof of (c) implies (d) such that $\underline{\mathrm{a}}=\Lambda e=\Lambda e \Lambda$ and $\underline{\mathrm{b}}=(1-e) \Lambda=\Lambda(1-e) \Lambda$, where $e \Lambda(1-e)=(0)$. It is straightforward to see that the morphism

$$
\varphi: \Lambda \rightarrow\left(\begin{array}{cc}
e \Lambda e & 0 \\
(1-e) \Lambda e & (1-e) \Lambda(1-e)
\end{array}\right)
$$

given by

$$
\varphi(x)=\left(\begin{array}{cc}
e x e & 0 \\
(1-e) x e & (1-e) x(1-e)
\end{array}\right)
$$

is an isomorphism.

Using the previous results, the next corollary follows directly.

Corollary 3.22. Suppose that $\underline{\mathrm{a}}^{2}=\underline{\mathrm{a}}$ and that $\Lambda / \underline{\mathrm{a}}$ is a projective $\Lambda$-module. Then the subbifunctor $F=F_{[G]} \cap F_{\left[G^{\prime}\right]}$ has enough injectives and enough projectives. In particular,

$$
\mathcal{I}(F)=\mathcal{I}(\Lambda) \cup D \operatorname{Tr}_{\Lambda}(\bmod \Lambda / \underline{\mathrm{a}}) \cup \bmod \Lambda / \underline{\mathrm{b}}
$$

and

$$
\mathcal{P}(F)=\mathcal{P}(\Lambda) \cup \bmod \Lambda / \underline{\mathrm{a}} \cup \operatorname{Tr}_{\Lambda} D(\bmod \Lambda / \underline{\mathrm{b}}) .
$$

\section{Applichtions to vector space Categories}

4.1. Representations of bimodules. In the study of algebras of finite or tame representation type we use various techniques of reduction to so-called subspace categories of vector space categories. A reduction of this form appeared in the work of Nazarova and Roiter on the Brauer-Thrall conjectures (see [NR]). A modified version called one-point extension was applied intensively by several authors (see e.g. [Ri1],[Ri2] as prominent examples) and is still one of the most efficient tools available. However, in the recent work of Gabriel et. al. [G-V] on the tame and wild dichotomy the original Nazarova-Roiter reduction technique was used again.

The aim of this section is to show that the results on relative homology developed in the previous sections can be used to understand the exact structures of the arising subspace categories in the cases just mentioned. This provides an interesting connection between relative homology and vector space category techniques, which seemed to be parts of the representation theory of artin algebras which lie very far from each other. We get an illustration of our earlier results, and can also apply them to obtain information on subspace categories. 
Before we recall the relevant material about vector space categories, we discuss bimodule problems more generally. Doing so, we will invoke various notations and basic results from [GR], but we remark that the use of bimodules has a long history (see e.g. [Dd]). We fix a commutative artinian ring $k$ and consider a Krull-Schmidt category $K$ that is is $k$-finite, which means by definition that $K$ is a $k$-additive category whose morphism spaces are finitely generated $k$-modules. For $k$-finite Krull-Schmidt categories which are skeletally small the notation is introduced in [G-V]. A bimodule over two skeletally small $k$-finite Krull-Schmidt categories $L$ and $K$ is a $k$-linear bifunctor $H: L \times K \rightarrow \bmod k$ which is covariant in the second and contravariant in the first argument. The category $\operatorname{rep}(H)$ of representations of $H$ has as objects the triples $(X, h, Y)$, where $X \in L, Y \in K$ and $h \in H(X, Y)$. A morphism from $(X, h, Y)$ to $\left(X^{\prime}, h^{\prime}, Y^{\prime}\right)$ is a pair $(f, g)$ of morphisms $f: X \rightarrow X^{\prime}$ in $L$ and $g: Y \rightarrow Y^{\prime}$ in $K$ such that $H(X, g)(h)=H\left(f, Y^{\prime}\right)\left(h^{\prime}\right)$. The category $\operatorname{rep}(H)$ is again a $k$-finite Krull-Schmidt category, and becomes an exact category in a canonical way by choosing as exact pairs those pairs of morphisms $\left((f, g),\left(f^{\prime}, g^{\prime}\right)\right)$ such that $\left(f, f^{\prime}\right)$ is a split exact pair in $L$ and $\left(g, g^{\prime}\right)$ is a split exact pair in $K$ (see $[\mathrm{G}-\mathrm{V}])$.

An example of a bimodule is given in the following way (see [GR, 9.1]): Consider an ideal $\underline{a}$ in an artin algebra $\Lambda$ over $k$. Denote by $G$ the corresponding left exact subfunctor $\operatorname{Hom}_{\Lambda}(\Lambda / \underline{\mathrm{a}}$,$) of the identity and by G^{\prime}$ the associated factor functor $\operatorname{id}_{\bmod \Lambda} / G$. Let $\underline{\mathrm{b}}$ be the ideal $G(\Lambda)$ of $\Lambda$. Then $H=\operatorname{Ext}_{\Lambda}^{1}($,$) is$ a bimodule over $\bmod \Lambda / \underline{\mathrm{b}}$ and $\bmod \Lambda / \underline{\mathrm{a}}$. The objects of $\operatorname{rep} H$ are the triples $(U, h, V)$, where $U$ is in $\bmod \Lambda / \underline{\mathrm{b}}$ and $V$ is in $\bmod \Lambda / \underline{\mathrm{a}}$ and $h \in \operatorname{Ext}_{\Lambda}^{1}(U, V)$ is an exact sequence $0 \rightarrow V \stackrel{f}{\longrightarrow} E \stackrel{g}{\longrightarrow} U \rightarrow 0$. We now give the relationship to our previous constructions. We first consider the relative theory for $\bmod \Lambda$ induced by the subfunctor $F=F_{[G]} \cap F_{\left[G^{\prime}\right]}$ of $\operatorname{Ext}_{\Lambda}^{1}($, $)$ in our previous notation. Then we define a functor $\Phi: \bmod \Lambda \rightarrow \operatorname{rep}(H)$ as follows. For an object $X$ in $\bmod \Lambda$, let $\Phi(X)=\left(G^{\prime}(X), h, G(X)\right)$, where $h$ is represented by the induced exact sequence $0 \rightarrow G(X) \stackrel{\iota X}{\longrightarrow} X \stackrel{\pi_{X}}{\longrightarrow} G^{\prime}(X) \rightarrow 0$. For a map $t: X \rightarrow X^{\prime}$, define $\Phi(t)=$ $\left(G^{\prime}(t), G(t)\right)$. Denoting as in Corollary 3.6 by $\mathfrak{R}$ the relation given by the maps $t: X \rightarrow X^{\prime}$, where $t=\iota_{X^{\prime}} s \pi_{X}$ for some $s: G^{\prime}(X) \rightarrow G\left(X^{\prime}\right)$, we see that a fully faithful functor $\bmod \Lambda / \mathfrak{R} \rightarrow \operatorname{rep} H$ is induced. The functor $\Phi: \bmod \Lambda \rightarrow \operatorname{rep}(H)$ is not necessarily dense. The image of $\Phi$ is the full subcategory $\operatorname{rep}_{\underline{a}} H$ of $\operatorname{rep} H$ whose objects are the triples $(U, h, V)$ with $f(V)=G(E)$, where $h$ is represented by $0 \rightarrow V \stackrel{f}{\longrightarrow} E \stackrel{g}{\longrightarrow} U \rightarrow 0$. The subcategory $\operatorname{rep}_{\mathrm{a}} H$ is clearly closed under extensions; that is, for each exact pair $(f: A \rightarrow B, g: B \rightarrow C)$ in rep $H$ with $A, C$ in $\operatorname{rep}_{\underline{a}} H$ also $B$ lies in repa $H$. Then an exact structure is induced from the exact structure on rep $H$ in the obvious way. We now have the following.

Proposition 4.1. Let the notations be as above. If we identify the category repa $\operatorname{rap}_{\mathbf{a}} H$ with $\bmod \Lambda / \mathfrak{R}$ via the functor $\Phi$, then the following hold.

(a) The exact structure on the category $\operatorname{rep}_{\underline{a}} H$ is obtained from mod $\Lambda$ in two steps, by first taking the exact structure on $\bmod \Lambda$ given by the subfunctor $F=$ $F_{[G]} \cap F_{\left[G^{\prime}\right]}$ of $\operatorname{Ext}_{\Lambda}^{1}($,$) , and then taking the induced exact structure on \bmod \Lambda / \Re$ for the relation $\mathfrak{R}$ defined above.

(b) The projective objects of $\operatorname{rep}_{\underline{\mathrm{a}}} H$ are the $\mathcal{E}_{F}$-projectives and the injective objects of rep $\operatorname{rep}_{\underline{a}} H$ are the $\mathcal{E}_{F}$-injectives.

(c) The category rep ra $_{H} H$ has almost split pairs. 
4.2. Subspace categories of vector space categories. We now recall the notions of a vector space category and its subspace category and motivate the concepts from the point of view of bimodules.

In many applications bimodules of a particularly simple shape arise. Namely, one considers the case that $L$ is a semisimple category with only one simple object $S$ (up to isomorphism). Let $D=\operatorname{End}_{\Lambda}(S)^{\text {op }}$. Then $D$ is a division ring which is finitely generated over $k$. In this situation the bifunctor $H$ is completely determined by the covariant functor $M:=H(S):, K \rightarrow \bmod D$. Moreover any object of $\operatorname{rep}(H)$ lying in $H\left(S^{n}, X\right)$ may obviously be rewritten as a sequence of $n$ elements in $H(S, X)=M(X)$. By formalizing this interpretation one encounters the concept of the subspace category of a vector space category: Namely, a (D-) vector space category is just a $k$-additive functor $M: K \rightarrow \bmod D$, where $K$ is a skeletally small $k$-finite Krull-Schmidt category. Its subspace category $M^{D}$ (again a $k$-finite KrullSchmidt category) has as objects the triples $X=\left(X_{\omega}, \gamma_{X}, X_{0}\right)$, where $X_{\omega} \in \bmod D$, $X_{0} \in K$ and $\gamma_{X} \in \operatorname{Hom}_{D}\left(X_{\omega}, M\left(X_{0}\right)\right)$. Morphisms $X \rightarrow X^{\prime}$ in this category are pairs $f=\left(f_{\omega}, f_{0}\right)$ such that $f_{\omega}: X_{\omega} \rightarrow X_{\omega}^{\prime}$ is $D$-linear, $f_{0}: X_{0} \rightarrow X_{0}^{\prime}$ is a morphism in $K$ and $M\left(f_{0}\right) \gamma_{X}=\gamma_{X^{\prime}} f_{\omega}$. The Krull-Schmidt category $M^{D}$ is equipped with an exact structure by using as exact pairs the pairs $(f, g)$ where $f: X \rightarrow Y$ and $g: Y \rightarrow Z$ are such that the sequences $\left(f_{0}, g_{0}\right)$ in $K$ and $\left(f_{\omega}, g_{\omega}\right)$ in $\bmod D$ are split exact, where the last condition is superfluous.

We now return to the example of the bimodule $H=\operatorname{Ext}_{\Lambda}^{1}($,$) discussed above.$ Assume that the ideal a giving rise to this bimodule is homogeneous and semisimple as a right $\Lambda$-module. Letting $\underline{\mathrm{b}}=G(\underline{\mathrm{a}})$ as before, $\bmod \Lambda / \underline{\mathrm{b}}$ is a semisimple category with only one simple object $S$ up to isomorphism. The associated bimodule interpreted as a vector space category is just $M=\operatorname{Ext}_{\Lambda}^{1}(S):, \bmod \Lambda / \underline{a} \rightarrow$ $\bmod D$, where $D=\operatorname{End}_{\Lambda}(S)^{\mathrm{op}}$. For the functor $\Phi: \bmod \Lambda \rightarrow M^{D}$ we have $\Phi(X)=\left(G^{\prime}(X), h, G(X)\right)$, where $h \in H=\operatorname{Ext}_{\Lambda}^{1}\left(G^{\prime}(X), G(X)\right)$ is the exact sequence $0 \rightarrow G(X) \stackrel{\iota_{X}}{\longrightarrow} X \stackrel{\pi_{X}}{\longrightarrow} G^{\prime}(X) \rightarrow 0$. This means that $h$ is the image of $\operatorname{id}_{G^{\prime}(X)}$ under the induced map $\operatorname{Hom}_{\Lambda}\left(G^{\prime}(X), G^{\prime}(X)\right) \rightarrow \operatorname{Ext}_{\Lambda}^{1}\left(G^{\prime}(X), G(X)\right)$. Consider now the induced map $p_{X}: \operatorname{Hom}_{\Lambda}\left(S, G^{\prime}(X)\right) \rightarrow \operatorname{Ext}_{\Lambda}^{1}(S, G(X))$. Since $G^{\prime}(X) \cong S^{n}$ for some $n, \operatorname{Hom}_{\Lambda}\left(S, G^{\prime}(X)\right.$ can be identified with the $D$-space $D^{n}$. Hence we have a functor $\Phi: \bmod \Lambda \rightarrow M^{D}$, where $\Phi(X)=\left(D^{n}, p_{X}, G(X)\right)$.

We finally want to identify the subcategory of $M^{D}$ corresponding to the subcategory repa $H$ of rep $H$. As observed in [GR], one has to distinguish two cases. If $\underline{\mathrm{a}} S \neq 0$, then $\operatorname{rep}_{\mathrm{a}}(H)=\operatorname{rep}(H)$. However, if $\underline{\mathrm{a}} S=0$, then $\operatorname{rep}_{\mathrm{a}}(H)$ corresponds to the subcategory $M_{N}^{D}$ of $M^{D}$, where $N$ denotes the subbifunctor $N=\operatorname{Ext}_{\Lambda / \underline{\mathrm{a}}}^{1}(S$, of $M$ and $M_{N}^{D}$ consists of all objects $X=\left(X_{\omega}, \gamma_{X}, X_{0}\right)$ with $\gamma_{X}^{-1}\left(N\left(X_{0}\right)\right)=0$.

Before we formulate the special case of our above proposition we observe that since all sequences in $\bmod D$ split we get $F_{[G]} \cap F_{\left[G^{\prime}\right]}=F_{[G]}=F_{\bmod \Lambda / \mathrm{a}}$. We use our results from section 3 to describe the projective and injective objects in the resulting subspace categories of vector space categories.

Proposition 4.2. Let $\Lambda$ be an artin algebra and a an ideal in $\Lambda$ which is homogeneous semisimple as a right $\Lambda$-module. Denote by $S$ the corresponding simple left $\Lambda$-module, and let $\mathcal{C}=M_{N}^{D}$ if $\underline{\mathrm{a}} S=0$ and $\mathcal{C}=M^{D}$ otherwise.

(a) The functor $\Phi: \bmod \Lambda \rightarrow \mathcal{C}$ induces an equivalence of categories between $\bmod \Lambda / \mathfrak{R}$ and $\mathcal{C}$. 
(b) If we equip $\bmod \Lambda$ with the exact structure given by the subfunctor $F_{\bmod \Lambda / \underline{\mathrm{a}}}$ and $\bmod \Lambda / \Re$ with the induced exact structure, then this equivalence maps the exact pairs in $\bmod \Lambda / \mathfrak{R}$ to the exact pairs in $\mathcal{C}$.

(c) The indecomposable projective objects in $\mathcal{C}$ are the images of the indecomposable objects in $\mathcal{P}(\Lambda)$ and in $\bmod \Lambda / \underline{\mathrm{a}}$, and the indecomposable injective objects are the images of the indecomposable modules in $\mathcal{I}(\Lambda)$ and in $D \operatorname{Tr}(\bmod \Lambda / \underline{\mathrm{a}})$.

(d) $\mathcal{C}$ has almost split pairs.

We can give a more explicit description of the projectives. Denote by $P(S)$ the projective cover of $S$. Obviously $P(S)$ is up to isomorphism the only indecomposable projective module in $\bmod \Lambda$ which is not a $\Lambda / \underline{a}$-module. On the other hand, if $X \in \bmod \Lambda / \underline{\mathrm{a}}$, then $\Phi(X)=(0,0, X)$. Consequently, we obtain

Corollary 4.3. The indecomposable projective objects in $\mathcal{C}$ are exactly all $(0,0, X)$ such that $X$ in $\bmod \Lambda / \underline{\mathrm{a}}$ is indecomposable together with the object $\left(D, \alpha_{S}, \operatorname{rad} P(S)\right)$, where $\alpha_{S}$ maps the unit element of $D$ to the canonical extension:

$$
0 \rightarrow \operatorname{rad} P(S) \longrightarrow P(S) \longrightarrow S \rightarrow 0 .
$$

We do not know a similar description for the injectives.

4.3. Special cases. Let us now apply our results to one-point extensions. Let $\Lambda=\left(\begin{array}{cc}D & 0 \\ R & B\end{array}\right)$, where $B$ is an artin algebra, $D$ is a division ring and $R$ a $B$ - $D$ bimodule. The objects of $\bmod \Lambda$ can then be described as triples $(X, f, Y)$, where $X$ is in $\bmod D, Y$ is in $\bmod B$ and $f: X \rightarrow \operatorname{Hom}_{B}(R, Y)$ is a $D$-linear map. In other words, the functor $M=\operatorname{Hom}_{B}(R):, \bmod B \rightarrow \bmod D$ is a vector space category. Hence $\bmod \Lambda$ can be identified with the subspace category $M^{D}$, but the exact structure introduced on $M^{D}$ above does not contain all short exact sequences in $\bmod \Lambda$ but only those sequences whose restrictions to $\bmod B$ split.

We want to show that this example fits into the construction discussed before. Let $\underline{\mathrm{a}}=\left(\begin{array}{cc}D & 0 \\ R & 0\end{array}\right)$. Then $\underline{\mathrm{a}}$ is an idempotent ideal of $\Lambda$ which is homogeneous semisimple as right $\Lambda$-module. We have $\underline{\mathrm{b}}=\left(\begin{array}{cc}0 & 0 \\ R & B\end{array}\right), \Lambda / \underline{\mathrm{a}} \cong B$ and $\Lambda / \underline{\mathrm{b}} \cong D$. Hence the associated simple left $\Lambda$-module $S$ can be identified with $D$. It is clear that the projective cover $P(S)$ of $S$ coincides with $\underline{\text { a }}$ as a left $\Lambda$-module and that $\operatorname{rad} P(S)=R$. The factor algebra $\Lambda / \underline{\mathrm{a}}$ can be identified with $B$. Since $\underline{\mathrm{a}} S \neq 0$ and $\mathfrak{R}$ is trivial, this gives an equivalence $\bmod \Lambda \rightarrow M^{D}$. Note that the functor $M=\operatorname{Hom}_{B}(R):, \bmod B \rightarrow \bmod D$ is naturally isomorphic to $\operatorname{Ext}_{\Lambda}^{1}(S$,$) .$

As follows from Corollary 4.3, the indecomposable projective objects in $M^{D}$ are $\left(D, \alpha_{S}, R\right)$ and the objects $(0,0, X)$, where $X$ is indecomposable in $\bmod B$. In order to use Proposition 4.2 to describe the injective objects, consider the full and faithful functor $\bmod B \rightarrow \bmod \Lambda$ given by sending an object $X$ in $\bmod B$ to $\bar{X}=\left(\operatorname{Hom}_{B}(R, X)\right.$, id, $\left.X\right)$. It is easy to see that for $X \in \bmod B$ we have $D \operatorname{Tr}_{\Lambda} X \cong$ $\overline{D \operatorname{Tr}_{B} X}$ (see [Ri2]). The indecomposable injective $\Lambda$-modules are $(D, 0,0)$ and $\bar{X}$, where $X$ is indecomposable injective in $\bmod B$. Hence the indecomposable injective objects in $\bmod \Lambda$ for the functor $F_{\bmod B}$ are $(D, 0,0)$ and $\bar{X}$ for $X$ indecomposable in $\bmod B$. Using the isomorphism $\operatorname{Hom}_{B}(R,) \cong \operatorname{Ext}_{\Lambda}^{1}(S):, \bmod B \rightarrow$ $\bmod D$, we see that the indecomposable injective objects in $M^{D}$ are $(D, 0,0)$ and $\left(\operatorname{Ext}_{\Lambda}^{1}(S, X)\right.$, id, $\left.X\right)$ for $X$ indecomposable in $\bmod B$. This description of the projective and injective objects in $M^{D}$ was also obtained in [Ri2]. 
We consider a small variation of the above example. We now let a be the ideal $\left(\begin{array}{ll}0 & 0 \\ R & 0\end{array}\right)$ in $\Lambda=\left(\begin{array}{cc}D & 0 \\ R & B\end{array}\right)$ which satisfies $\underline{\mathrm{a}}^{2}=0$. This ideal $\underline{\mathrm{a}}$ is also homogeneous semisimple as right $\Lambda$-module, and the corresponding simple module $S$ is the same as before. But now the factor algebra $\Lambda / \underline{\mathrm{a}}$ is $D \times B$. Hence the indecomposable $\Lambda / \underline{a}$-modules are not only the indecomposable $B$-modules but also $S$ itself appears. Thus, in contrast to the previous case, $\mathcal{C}$ is properly contained in $M^{D}$ although $N=0$. Actually, the only indecomposable object in $M^{D}$ not belonging to $M_{N}^{D}$ is $(D, 0,0)$. Altogether, we find an additional indecomposable projective object, namely $(0,0, S)$, and lose the indecomposable injective object $(D, 0,0)$. However, if $R \neq 0$, then $S$ is not projective and hence a new indecomposable injective object, namely $\Phi\left(D \operatorname{Tr}_{\Lambda} S\right)$, arises. We do not know a good description for this subspace.

Let us finally come to the classical reduction functor of Roiter, which is the functor $\Phi$ if we consider the following situation: The given algebra $\Lambda$ is defined over an algebraically closed field and is not semisimple. Therefore one can choose an element which generates an ideal a lying in $\operatorname{rad} \Lambda$ which is simple as right and left module. Consequently, $S$ is a $\Lambda$ a -module and $\mathcal{C}=M_{N}^{D}$. The general description of the projectives as in our remark applies. Concerning the category of injectives in $M_{N}^{D}$, we only know that it is $\Phi(\mathcal{I}(\Lambda)) \cup \Phi(D \operatorname{Tr}(\bmod \Lambda / \underline{a}))$. This is not a very convenient description, but at least it can be calculated in some examples.

\section{An eXAmple FOR NON-EXISTEnCE OF Almost SPlit SEQUENCES}

5.1. Prinjective modules. We saw in the previous section that the categories $M^{D}$ and $M_{N}^{D}$ do have almost split sequences provided they are obtained from module categories via reduction functors. For $M^{D}$ the existence of almost split sequences is known if $M$ satisfies certain finiteness conditions, which are always satisfied in case $K$ has only finitely many isomorphism classes of indecomposable objects (see e.g. [GR]). The purpose of this section is to present an example where this fails for $M_{N}^{D}$.

We suppose that $K$ is a $k$-finite Krull-Schmidt category over an algebraically closed field $k$, and consequently $D=k$, so that $M: K \rightarrow \bmod k$. To construct the example, we first recall how to realize the category $M_{N}^{k}$ as extension-closed full subcategory of the category of modules over a finite-dimensional $k$-algebra. Since $K$ is supposed to have only finitely many isomorphism classes of indecomposable objects, we may identify $K$ with the category $\operatorname{proj} A$ of finite-dimensional projective left $A$-modules, where the basic algebra $A$ is the endomorphism algebra of a minimal additive generator of $K$. Consequently $M$ transforms into a tensor functor with a right $A$-module which by abuse of language we denote by $M$ as well. Thus the objects of $M^{k}$ are of the shape $\left(X_{\omega}, \gamma_{X}, X_{0}\right)$ such that $X_{\omega} \in \bmod k, X_{0} \in \operatorname{proj} A$ and $\gamma_{X} \in \operatorname{Hom}_{k}\left(V_{\omega}, M \otimes_{A} X_{0}\right)$.

We define the $k$-algebra $B$ as the triangular matrix algebra $\left(\begin{array}{cc}A & 0 \\ M & k\end{array}\right)$, which is usually called the one-point coextension of $A$ by $M$. The finite-dimensional left modules $U$ over $B$ may be considered as triples $U=\left(U_{0}, \mu_{U}, U_{1}\right)$ such that $U_{0} \in \bmod A, U_{1} \in \bmod k$ and $\mu \in \operatorname{Hom}_{k}\left(M \otimes_{A} U_{0}, U_{1}\right)$. Namely, the corresponding module is $U_{0} \oplus U_{1}$, the multiplication by elements of $A$ on $U_{0}$ and by elements of $k$ on $U_{1}$ is obvious, and the multiplication of an element $m \in M$ by $u_{0} \in U_{0}$ is given as $m \cdot u_{0}=\mu_{U}\left(m \otimes u_{0}\right) \in U_{1}$. Morphisms in $\bmod B$ appear as pairs $\left(f_{0}, f_{1}\right)$ with the obvious commutativity requirements. 
The full subcategory prin $B$ of $\bmod B$ given by all $U=\left(U_{0}, \mu_{U}, U_{1}\right)$ such that $U_{0} \in \operatorname{proj} A$ is easily seen to be extension closed. It is often called the subcategory of prinjective $B$-modules. The module $S:=(0,0, k)$ is simple projective and lies in prin $B$. The full subcategory $\operatorname{prin}^{\prime} B$ of prin $B$ given by all objects which do not admit a direct summand isomorphic to $S$ is therefore again extension closed. We will describe an exact equivalence $\Theta: M_{0}^{k} \rightarrow \operatorname{prin}^{\prime} B$. For this purpose we pick an object $X=\left(X_{\omega}, \gamma_{X}, X_{0}\right)$ in $M_{0}^{k}$ and denote by $\hat{\gamma}_{X}$ a cokernel map $M \otimes_{A} X_{0} \rightarrow \hat{X}_{\omega}$ of $\gamma_{X}$. The equivalence $\Theta$ sends the object $X$ to the module $\left(X_{0}, \hat{\gamma}_{X}, \hat{X}_{\omega}\right)$ and acts on the morphisms in the obvious way.

If $P_{i}, i=1, \ldots, n$, is a complete family of non-isomorphic indecomposable modules in $\operatorname{proj} A$, then $S$ together with $\left(P_{i}\right.$, id, $\left.M \otimes_{A} P_{i}\right), i=1, \ldots, n$, are consequently up to isomorphism the only indecomposable objects in $\operatorname{proj} B$. As $\operatorname{proj} B \subseteq \operatorname{prin} B$ and prin $B$ is closed under kernels of epimorphisms, $\operatorname{proj} B$ is the category of projectives in $\operatorname{prin} B$.

5.2. Modules with projective restriction. If $N$ is any submodule of $M$, then the algebra $B_{N}:=\left(\begin{array}{cc}A & 0 \\ N & k\end{array}\right)$ may be considered as a subalgebra of $B=B_{M}$ in the obvious way. This inclusion gives rise to an exact restriction functor $\bmod B \rightarrow$ $\bmod B_{N}$. We denote by $\operatorname{prin}_{N} B$ the full subcategory of $\bmod B$ given by the modules with projective restriction to $B_{N}$. Clearly we have $\operatorname{proj} B=\operatorname{prin}_{M} B \subseteq \operatorname{prin}_{N} B \subseteq$ $\operatorname{prin}_{0} B=\operatorname{prin} B$.

Now an object $X \in M_{0}^{k}$ lies in $M_{N}^{k}$ iff the induced map $\hat{\gamma}_{X}: N \otimes_{A} X_{0} \rightarrow \hat{X}_{\omega}$ is injective, which in turn means that $\Theta(X) \in \operatorname{prin}_{N} B$. This shows that $\Theta$ furnishes an equivalence $M_{N}^{k} \rightarrow \operatorname{prin}_{N}^{\prime} B$, where $\operatorname{prin}_{N}^{\prime} B$ is of course defined as the full subcategory of $\operatorname{prin}_{N} B$ given by modules which do not admit a direct summand isomorphic to $S$. We will continue by studying $\operatorname{prin}_{N} B$ in a specific example.

5.3. The example. We put $K:=\bmod k$ and consider the functor

$$
M=M \otimes_{k}-: \bmod k \rightarrow \bmod k,
$$

where $M$ is an $r$-dimensional vector space over $k$ for some natural number $r$. We see that $A=k$ and $B_{r}:=B$ is the $r$-Kronecker algebra which is the path algebra over $k$ of the quiver $\Delta_{r}$ shown below (the arrows from the vertex 0 to the vertex 1 correspond to a chosen basis $\alpha_{1}, \ldots, \alpha_{r}$ of $M$ ):

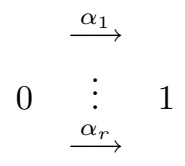

Hence we may identify modules $U=\left(U_{0}, \mu_{U}, U_{1}\right)$ with representations of the quiver $\Delta_{r}$ i.e. with tuples $\left(U_{0}, U\left(\alpha_{1}\right), \ldots, U\left(\alpha_{r}\right), U_{1}\right)$, where the linear maps $U\left(\alpha_{i}\right): U_{0} \rightarrow$ $U_{1}$ are defined by $U\left(\alpha_{i}\right)\left(u_{0}\right)=\mu_{U}\left(\alpha_{i} \otimes u_{0}\right)$ for all $u_{0} \in U_{0}$. The vector $\operatorname{dim} U:=$ $\left(\operatorname{dim}_{k} U_{0}, \operatorname{dim}_{k} U_{1}\right)$ is called the dimension vector of $U$.

Since $A$ semisimple, the categories $\bmod B_{r}$ and prin $B_{r}$ coincide. If $N$ is a submodule of $M$, then by adapting bases we may assume that $N$ is generated by $\alpha_{1}, \ldots, \alpha_{q}$ for some $0 \leq q \leq r$. Hence we get $\left(B_{r}\right)_{N}=B_{q}$. Because $B_{q}$ is hereditary, the subcategory $\operatorname{prin}_{N} B$ is closed under submodules. Consequently $\operatorname{prin}_{N} B$ is the torsion free class $\mathcal{F}$ of a torsion theory $(\mathcal{T}, \mathcal{F})$. 
Let us consider the various cases for $r$ and $q$. If $q=0$, then $N=0$, and, if $q=r$, then $N=M$. Hence these cases were already considered above. Thus we may suppose $r>1$ and $0<q<r$.

For $r>1$ the Auslander-Reiten quiver of $B_{r}$ always has one preprojective component, one preinjective component and an infinite family of regular components. Clearly the projective modules are in $\mathcal{F}$. We obtain another torsion free indecomposable module if we consider the unique non-simple indecomposable projective $B_{q}$-module $V$ as $B_{r}$-module via extension by 0 . As $r>1$ and $q<r$, the module $V$ is regular. If the preprojective component contains an indecomposable which is not torsion free, then it contains also an indecomposable torsion module. Namely, because the preprojective component is closed under predecessors, any direct summand of the torsion submodule has to be preprojective as well. But any preprojective module generates all regular modules, and therefore $V$ would be torsion. From this contradiction we derive that all preprojective modules lie in $\mathcal{F}$.

To deal with the preinjective component, we observe that for $r>1$ all preinjective modules $U$ satisfy $\operatorname{dim}_{k} U_{0}>\operatorname{dim}_{k} U_{1}$ whereas all projective $B_{q}$-modules $U$ satisfy $\operatorname{dim}_{k} U_{0} \leq \operatorname{dim}_{k} U_{1}$ for all $q \geq 0$. Therefore none of the preinjective modules can lie in $\mathcal{F}$. We claim that actually all preinjective modules belong to $\mathcal{T}$. The reason is that the preinjective component is closed under successors and therefore any indecomposable summand of the torsion free factor module of a non-torsion module in the preinjective component would have to be preinjective as well.

For $r=2$ only the case $q=1$ is left to consider. The regular components form a family of homogeneous tubes indexed over the projective line $\mathbb{P}^{1}(k)$. As $\operatorname{prin}_{N} B$ is closed under extensions and submodules, such a tube lies in $\mathcal{F}=\operatorname{prin}_{N} B_{2}$ if and only if the simple regular module at the mouth of the tube lies in $\operatorname{prin}_{N} B_{2}$. For $(a: b) \in \mathbb{P}^{1}(k)$ this simple regular module is just $(k, a, b, k)$, which lies in $\operatorname{prin}_{N} B_{2}$ if and only if $a \neq 0$. Consequently all the tubes with exception of the one corresponding to $(0: 1) \in \mathbb{P}^{1}(k)$ lie in $\operatorname{prin}_{N} B_{2}$. Note that $\operatorname{prin}_{N} B$ does not have any injective objects but obviously has almost split sequences. For completeness we remark that the tube associated with $(0: 1)$ consists entirely of torsion modules, and consequently the torsion theory $(\mathcal{T}, \mathcal{F})$ splits.

It remains to deal with the case $r>2$, in which $B_{r}$ is of wild representation type. Now all the regular components are of the shape $\mathbb{Z A}_{\infty}$. The modules in the $D \operatorname{Tr}$ orbit forming the rim of such a component are called quasi-simple. Since we already know that all preprojective modules are in $\mathcal{F}$ and all preinjective modules are in $\mathcal{T}$, we can invoke [AK, Corollary 5.2]. By this result there are three possibilities. The first two are that all regular modules are torsion or all regular modules are torsion free. We already saw above that there is a torsion free regular module. On the other hand, for $r>2$ the extension by 0 of the non-simple indecomposable injective $B_{r-1}$-module is regular and not in $\mathcal{F}$, as already observed. Hence only the third alternative of [AK, Corollary 5.2] can be valid, which says that in each regular component $\mathcal{C}$ there exist quasi-simple modules $U_{\mathcal{C}}$ and $V_{\mathcal{C}}$ such that the successors of $U_{\mathcal{C}}$ in $\mathcal{C}$ belong to $\mathcal{F}=\operatorname{prin}_{N} B_{r}$ and the predecessors of $V_{\mathcal{C}}$ in $\mathcal{C}$ belong to $\mathcal{T}$. From this follows that each regular component contains an indecomposable module which is neither torsion nor torsion free. Hence the torsion theory $(\mathcal{T}, \mathcal{F})$ is not splitting.

We want to show that, after possibly changing $U_{\mathcal{C}}$ and $V_{\mathcal{C}}$, the modules in $\mathcal{C}$ belonging to $\mathcal{F}=\operatorname{prin}_{N} B_{r}$ are precisely the successors of $U_{\mathcal{C}}$ in $\mathcal{C}$ and the modules in $\mathcal{C}$ belonging to $\mathcal{T}$ are precisely the predecessors of $V_{\mathcal{C}}$ in $\mathcal{C}$. For $\mathcal{F}=\operatorname{prin}_{N} B_{r}$ this follows from the following lemma. 
Lemma 5.1. If $X$ is an indecomposable non-injective $B_{r}$-module which lies in $\operatorname{prin}_{N} B_{r}$, then $\operatorname{Tr} D X$ also lies in $\operatorname{prin}_{N} B_{r}$.

Proof. That $X$ lies in $\mathcal{F}$ implies that the intersection of the kernels of the maps $X\left(\alpha_{1}\right), \ldots, X\left(\alpha_{q}\right)$ is zero. For the right $B_{r}$-module $Y=D(X)$ considered as representation of the opposite quiver $\Delta_{r}^{*}$ this means that the sum of the images of the maps $Y\left(\alpha_{1}^{*}\right), \ldots, Y\left(\alpha_{q}^{*}\right)$ is $Y_{0}$.

Let us denote by $P_{0}, P_{1}$ the indecomposable left and by $P_{0}^{*}, P_{1}^{*}$ the indecomposable right projective $B_{r}$-modules. If

$$
0 \rightarrow\left(P_{0}^{*}\right)^{n} \stackrel{p^{*}}{\longrightarrow}\left(P_{1}^{*}\right)^{m} \longrightarrow Y \rightarrow 0
$$

is a minimal projective presentation of $Y$, then $p^{*}$ is given by a left multiplication by an $m \times n$ matrix $\Gamma$ with entries $\gamma_{\nu \mu}$ in $M$. Hence each entry can be written as $\gamma_{\nu \mu}=\sum_{i=1}^{r} \gamma_{\nu \mu}^{(i)} \alpha_{i}$ with $\gamma_{\nu \mu}^{(i)}$ in $k$. For $i=1, \ldots, r$ we denote by $\Gamma_{i}$ the $m \times n$ matrix over $k$ with coefficients $\gamma_{\nu \mu}^{(i)}$.

The exactness of the above projective presentation together with the fact that the sum of the images of the maps $Y\left(\alpha_{1}^{*}\right), \ldots, Y\left(\alpha_{q}^{*}\right)$ is $Y_{0}$ yields that the $(r-q) m \times n$ matrix

$$
\left(\begin{array}{c}
\Gamma_{q+1} \\
\vdots \\
\Gamma_{r}
\end{array}\right)
$$

over $k$ has rank $(r-q) m$. In particular, the matrix $\Gamma_{q}$ has rank $m$. The projective presentation of $\operatorname{Tr} Y=D \operatorname{Tr} X$ is given by

$$
0 \rightarrow P_{1}^{m} \stackrel{p}{\longrightarrow} P_{0}^{n} \longrightarrow D \operatorname{Tr} X \rightarrow 0
$$

where $p$ is given by the right multiplication $\Gamma$. That $\Gamma_{q}$ has rank $m$ shows that the restriction of $D \operatorname{Tr} X$ to $B_{r-1}$ is projective. Consequently any restriction to some $B_{q}, 1 \leq q \leq r-1$, is projective, and therefore $D \operatorname{Tr} X$ lies in $\operatorname{prin}_{N} B_{r}$.

Note that again there are no injectives in $\operatorname{prin}_{N} B_{r}$, which by [AK, Theorem (B)] gives also the existence of a module $V_{\mathcal{C}}$ as desired.

Let us finally study almost split sequences in $\operatorname{prin}_{N} B_{r}$. Obviously for every indecomposable module $U$ in $\operatorname{prin}_{N} B_{r}$ there is an almost split sequence starting in $U$. We claim that for every regular component $\mathcal{C}$ and every module $V$ in the ray starting in $U_{\mathcal{C}}$ there does not exist a minimal right almost split map $h: W \rightarrow V$ in $\operatorname{prin}_{N} B_{r}$. Since $\operatorname{Hom}_{B_{r}}(Z, S)=0$ for all indecomposable $B_{r}$-modules which are not isomorphic to $S$, there also does not exist a right almost split map $h: W^{\prime} \rightarrow V$ in $\operatorname{prin}_{N}^{\prime} B_{r}$.

To prove our claim by contradiction, we assume that a right minimal $h: W \rightarrow V$ in $\operatorname{prin}_{N} B_{r}$ exists. First, we know that $V$ is not projective and therefore there is a non-split short exact sequence in $\operatorname{prin}_{N} B_{r}$ ending in $V$. By factoring the nonsplit epimorphism ending in $V$ through $h$, we see that $h$ has to be an epimorphism as well. Since $\operatorname{prin}_{N} B_{r}$ is closed under submodules, this yields an almost split sequence ending in $V$ and starting in some $V^{\prime}$. But we know the almost split sequences starting in all indecomposables in $\bmod B_{r}$, and $V$ never occurs as end term.

We remark that as a torsion free class the subcategory $\operatorname{prin}_{N} B_{r}$ is covariantly finite in $\bmod B_{r}$. Hence the one-sided existence of almost split sequences also follows from $[\mathrm{Kl}]$. 


\title{
APPENDIX
}

\section{On GABRIEL-RoIter's AXIOMS FOR EXACT CATEGORIES}

\begin{abstract}
We give a direct proof of the fact that the Gabriel-Roiter axioms characterize those exact categories in the sense of Quillen in which all retractions have kernels.
\end{abstract}

\section{A. The STATEMEnt}

Recall the setting of [GR, Ch. 9] : Let $\mathcal{A}$ be an additive category. A pair $(i, d)$ of composable morphisms

$$
X \stackrel{i}{\rightarrow} Y \stackrel{d}{\rightarrow} Z
$$

is exact if $i$ is a kernel of $d$ and $d$ a cokernel of $i$. Let $\mathcal{E}$ be a class of exact pairs closed under isomorphism. By definition, a deflation is a morphism which appears as the second component of a conflation $(i, d) \in \mathcal{E}$. The first components $i$ are called inflations. Consider the following two sets of axioms.

Set 1 [GR, Ch. 9]

E1 The composition of two deflations is a deflation.

E2 For each $f \in \mathcal{A}\left(Z^{\prime}, Z\right)$ and each deflation $d \in \mathcal{A}(Y, Z)$, there is a $g \in$ $\mathcal{A}\left(Y^{\prime}, Y\right)$ and a deflation $d^{\prime}: Y^{\prime} \rightarrow \mathbf{Z}^{\prime}$ such that $d g=f d^{\prime}$.

E3 Identities are deflations; if $d e$ is a deflation, then so is $d$.

E3 ${ }^{\text {op }}$ Identities are inflations; if $j i$ is an inflation, then so is $i$.

Set $2[K$, App. A]

Ex0 The identity morphism of the zero object $1_{0}$ is a deflation.

Ex1 The composition of two deflations is a deflation.

Ex2 For each $f \in \mathcal{A}\left(Z^{\prime}, Z\right)$ and each deflation $d \in \mathcal{A}(Y, Z)$, there is a cartesian square (a pullback)

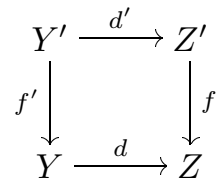

where $d^{\prime}$ is a deflation.

Ex2 ${ }^{\text {op }}$ For each $f \in \mathcal{A}\left(X, X^{\prime}\right)$ and each inflation $i \in \mathcal{A}(X, Y)$, there is a cocartesian square (a pushout)

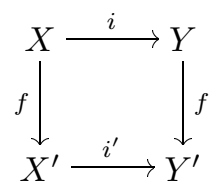

where $i^{\prime}$ is an inflation.

It is shown in $[\mathrm{K}$, App. A] that the axioms of Set 2 characterize the exact categories in the sense of Quillen $[\mathrm{Q}]$ and that each small category $(\mathcal{A}, \mathcal{E})$ satisfying the axioms of Set 2 admits a fully faithful and exact embedding $F: \mathcal{A} \rightarrow \mathcal{B}$ into an abelian category in such a way that a sequence of objects of $\mathcal{A}$ is in $\mathcal{E}$ iff its image under $F$ is short exact in $\mathcal{B}$. 
Proposition. The pair $(\mathcal{A}, \mathcal{E})$ satisfies the axioms of Set 1 iff if satisfies the axioms of Set 2 and retractions of $\mathcal{A}$ admit kernels.

The proposition will be proved in the following two sections.

\section{B. From Set 1 to Set 2}

Suppose that the axioms of Set 1 hold for $(\mathcal{A}, \mathcal{E})$. If we have a retraction $r$ of $\mathcal{A}$ admitting a section $s$, then $r s$ is an identity morphism. So $r s$ is a deflation by the first part of E3, and $r$ is a deflation by the second part of E3 again. So $r$ admits a kernel. Moreover, axiom Ex0 holds by E3 and axiom Ex1 holds by E1. To prove Ex2, suppose that we are given $f \in \mathcal{A}\left(Z^{\prime}, Z\right)$ and a deflation $d \in \mathcal{A}(Y, Z)$. The composition of

$$
p=\left[\begin{array}{l}
f \\
d
\end{array}\right]: Z^{\prime} \oplus Y \rightarrow \mathbf{Z}
$$

with the inclusion of the summand $Y$ is the deflation $d$. Hence, by axiom E3, the morphism $p$ is a deflation. Therefore $p$ admits a kernel and we have a pullback diagram

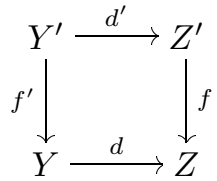

It remains to be shown that $d^{\prime}$ is a deflation. For this we choose any commutative square

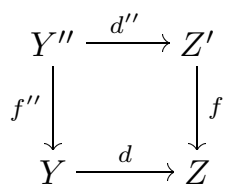

where $d^{\prime \prime}$ is a deflation. Indeed, such squares exist by axiom E2. Now by the pullback property, the morphism $d^{\prime \prime}$ factors as $d^{\prime} e$ for some morphism $e: Y^{\prime \prime} \rightarrow Y^{\prime}$. So $d^{\prime}$ is a deflation by axiom E3. This proves Ex2.

Axiom Ex $2^{\mathrm{op}}$ is harder to prove since Set 1 does not contain $\mathrm{E} 2^{\mathrm{op}}$. We proceed in several steps.

1st step: If the rows of a diagram

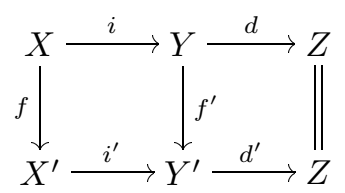

are conflations, then the left hand square is a conflation square (i.e. the sequence

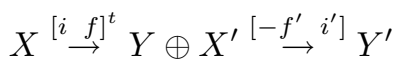

is a conflation). Indeed, first of all, it is easy to check that the left hand square is a pullback square, so that $\left[\begin{array}{ll}i & f\end{array}\right]^{t}$ is a kernel of $\left[\begin{array}{ll}-f^{\prime} & i^{\prime}\end{array}\right]$. It remains to be shown that 


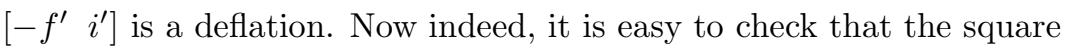

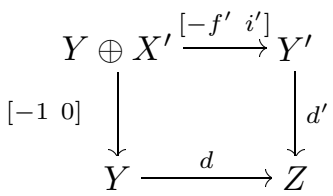

is a pullback. So $\left[\begin{array}{ll}-f^{\prime} & i^{\prime}\end{array}\right]$ is a conflation by $\operatorname{Ex} 2$ (which we have already proved to hold).

2nd step: In a diagram

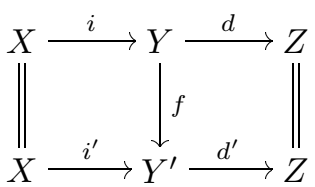

whose rows are conflations, the morphism $f$ is an isomorphism. Indeed, by the first step, the square

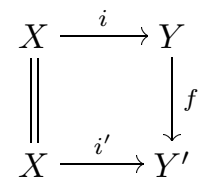

is a pushout (since it is even a conflation square).

3rd step: Axiom Ex2 ${ }^{\mathrm{op}}$ holds. Indeed, let a diagram

$$
X^{\prime} \stackrel{f}{\leftarrow} X \stackrel{i}{\rightarrow} Y
$$

with an inflation $i$ be given. The composition of the morphism

$$
j=\left[\begin{array}{l}
i \\
f
\end{array}\right]: X \rightarrow Y \oplus X^{\prime}
$$

with the projection onto $Y$ equals $i$. So by $\mathrm{E} 3^{\mathrm{op}}$, the morphism $j$ is an inflation. So we have a conflation square

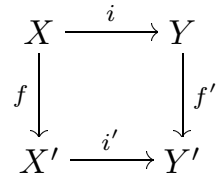

It remains to be shown that $i^{\prime}$ is an inflation. Form a conflation

$$
X \stackrel{i}{\rightarrow} Y \stackrel{p}{\rightarrow} Z .
$$

By the pushout property of the square, the morphism $p$ uniquely factors as $p^{\prime} f^{\prime}$ in such a way that $p^{\prime} i^{\prime}=0$. So we have a diagram

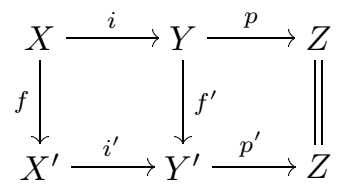

Here the morphism $p^{\prime}$ is a deflation by E3. Moreover, using the pushout property of the square, it is easy to check that $p^{\prime}$ is a cokernel of $i^{\prime}$. Unfortunately, this does 
not yet imply that $i^{\prime}$ is a kernel of $p^{\prime}$. But anyway, let $i^{\prime \prime}: X^{\prime \prime} \rightarrow Y^{\prime}$ be a kernel of $p^{\prime}$. Then the morphism $i^{\prime}$ factors as $i^{\prime \prime} g$ for some $g: X^{\prime} \rightarrow X^{\prime \prime}$. So we have a commutative diagram

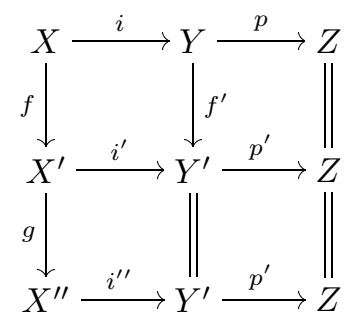

whose first and last rows are conflations. By looking at the squares on the left, we write down the following commutative diagram:

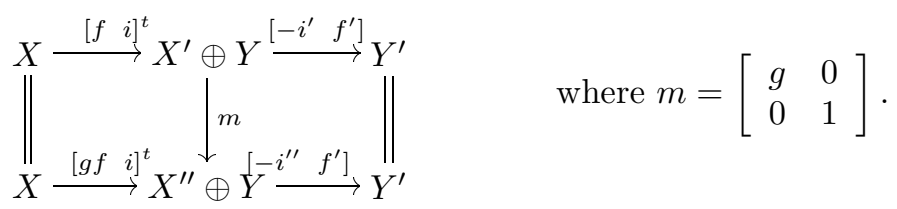

Its first row is a conflation by construction. Its second row is a conflation by the 1st step. So the morphism $m$ is invertible by the 2 nd step. Thus $g$ is an isomorphism and $i^{\prime}$ is an inflation.

\section{From Set 2 to Set 1}

Axiom E1 is Ex1. Axiom E2 is implied by Ex2. The first part of axiom E3 follows from Ex2 by the following pullback diagram:

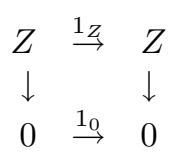

To prove the second part, suppose that we have a conflation

$$
X \stackrel{i}{\rightarrow} Y \stackrel{d e}{\rightarrow} Z .
$$

Then using Ex2 we form the pullback square

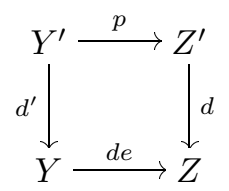

By the pullback property, there is a unique morphism $s: Y \rightarrow Y^{\prime}$ such that $d^{\prime} s=1_{Y}$ and $p s=e$. So $d^{\prime}$ is a retraction. And by hypothesis, $d^{\prime}$ admits a kernel $j: U \rightarrow Y^{\prime}$. It is not hard to check that $p j: U \rightarrow Z^{\prime}$ is a kernel of $d$. The fact that under these circumstances $d$ is a deflation is proved in [K, App. A] (this is exactly the content of Quillen's redundant axiom c).

Dually, the first part of axiom E3 ${ }^{\text {op }}$ follows from Ex0 and Ex $2^{\mathrm{op}}$. For the second part, we first prove that sections of $\mathcal{A}$ admit cokernels. Indeed, let $i: X \rightarrow Y$ and 
$r: Y \rightarrow X$ be such that $r i=1_{X}$. By the assumption, the morphism $r$ admits a kernel $s: Z \rightarrow Y$. The endomorphism $1_{Y}-i r$ of $Y$ satisfies $r\left(1_{Y}-i r\right)=0$. So we have $1_{Y}-i r=s d$ for some morphism $d: Y \rightarrow Z$. It is easy to check that $d$ is a cokernel of $i$.

Now we can dualize the proof of $\mathrm{E} 3$ to obtain $\mathrm{E} 3^{\mathrm{op}}$ (using the proof of axiom $\mathrm{c}^{\mathrm{op}}$ in $[\mathrm{K}$, App. A]).

\section{REFERENCES TO APPENDIX}

[GR] P. Gabriel, A. Roiter, Representations of finite-dimensional algebras, Encyclopaedia of the Mathematical Sciences, Vol. 73, A. I. Kostrikin and I. V. Shafarevich (Eds.), Algebra VIII, Springer 1992. MR 94h:16001

[K] B. Keller, Chain complexes and stable categories, Manus. Math. 67 (1990), 379-417. MR 91h:18006

[Q] D. Quillen, Higher Algebraic K-theory I, Springer LNM 341 (1973), 85-147. MR 49:2895

\section{REFERENCES}

[AK] Assem, I., Kerner, O., Constructing torsion pairs, J. Algebra 185 (1996), 19-41. MR 97f: 16024

$[\mathrm{Au}]$ Auslander, M., Functors and morphisms determined by objects, in Representations theory of artin algebras, Proceedings of the Philadelphia conference, Lecture Notes in Pure and Appl. Math., vol. 37, Dekker, New York, 1978, 1-244. MR 58:844

[AR] Auslander, M., Reiten, I., Homologically finite subcategories, Proceedings of Representations of algebras and related topics (Tsukuba, 1990), 1-42, London Math. Soc. Lecture Note Ser., 168, Cambridge Univ. Press, Cambridge, 1992. MR 94d:16017

[ARS] Auslander, M., Reiten, I., Smalø, S. O., Representation theory of artin algebras, Cambridge studies in advanced mathematics, vol. 36, Cambridge University Press, 1995. MR 96c: 16015

[ASm] Auslander, M., Smalø, S.O., Almost split sequences in subcategories, J. Algebra 96 (1981), 426-454. MR 82j:16048a

[ASo] Auslander, M., Solberg, Ø., Relative homology and representation theory I, Relative homology and homologically finite subcategories, Comm. in Alg., 21(9) (1993), 2995-3031. MR 94k:16010a

[BH] Butler, M. C. R., Horrocks, G., Classes of extensions and resolutions, Phil. Trans. Royal Soc., London, Ser. A, 254 (1961), 155-222. MR 32:5706

[Dd] Drozd, Yu.A., Matrix problems and categories of matrices, Zap. Nauchn. Sem. LOMI 28 (1972), 144-153; English transl., J. Soviet Math. 3 (1975), 692-699. MR 49:5037

[GR] Gabriel, P., Roiter, A., Representations of finite-dimensional algebras, Encyclopedia of the Mathematical Sciences, Vol. 73, A. I. Kostrikin and I. V. Shafarervich (Eds.), Algebra VIII, Springer 1992. MR 94h:16001b

[G-V] Gabriel, P., Nazarova, L.A., Roiter, A.V, Sergejchuk, V.V., Vossieck, D., Tame and wild subspace problems, Ukr. Math. J. 45 (1993), 313-352; English transl., Ukrainian Math. J. 45 (1993), 335-372. MR 94b:16012

[Ke1] Keller, B., Chain complexes and stable categories, Manuscripta Math. 67 (1990), 379-417. MR 91h:18006

[Ke2] Keller, B., On Gabriel-Roiter's axioms for exact categories, appendix to this paper.

[Kl] Kleiner, M., Approximations and almost split sequences in homologically finite subcategories, J. Algebra 198 (1997), 135-163. CMP 98:04

[NR] Nazarova, L.A., Roiter, A.V., Kategorielle Matrizenprobleme und die Brauer-ThrallVermutung, Mitt. Math. Sem. Giessen 115 (1975). MR 58:5790

[PS] de la Peña, J.A., Simson, D., Prinjective modules, reflection functors, quadratic forms and Auslander-Reiten sequences, Trans. Amer. Math. Soc. 329 (1992), 733-753. MR 9e:16005

[Qu] Quillen, D., Higher algebraic K-theory I, Springer LNM 341 (1973), 85-147. MR 49:2895 
[Ri1] Ringel, C.M., On algorithms for solving vector space problems II: Tame algebras, Lecture Notes in Math. 831 (1980), 137-287. MR 82j:16056

[Ri2] Ringel, C.M., Tame algebras and integral quadratic forms, Lecture Notes in Math. 1099, 1984. MR 87f: 16027

Fakultät für Mathematik, Universität Bielefeld, Postfach 100131, D-33501 BieleFELD, Germany

E-mail address: draexler@mathematik.uni-bielefeld.de

INSTITUTT FOR MATEMATIKK OG STATISTIKK, NORGES TEKNISK-NATURVITENSKAPELIGE UNIVERSITET, N-7055 DragVoll, NorWay

E-mail address: idunr@matsh.ntnu.no

InSTITUTT FOR MATEMATIKK OG STATISTIKK, NORGES TEKNISK-NATURVITENSKAPELIGE UNIVERSiteT, N-7055 Dragvoll, NorWay

E-mail address: sverresm@math.ntnu.no

InSTITUTT FOR MATEMATIKK OG STATISTIKK, NORGES TEKNISK-NATURVITENSKAPELIGE UNIVERSitet, N-7055 Dragvoll, Norway

E-mail address: oyvinso@math.ntnu.no

U.F.R. De Mathématiques, U.R.A. 748 du CNRS, Université Paris 7, 2, place Jussieu, 75251 Paris Cedex 05, France

E-mail address: keller@math.jussieu.fr 